\title{
The Effect of 3D Transport-induced Disequilibrium Carbon Chemistry on the Atmospheric Structure, Phase Curves, and Emission Spectra of Hot Jupiter HD 189733b
}

\author{
Maria E. Steinrueck ${ }^{1}$ (1), Vivien Parmentier ${ }^{2}$ (i), Adam P. Showman ${ }^{1}$, Joshua D. Lothringer ${ }^{1}$ (i), and Roxana E. Lupu ${ }^{3}$ (1) \\ ${ }^{1}$ Lunar and Planetary Laboratory, University of Arizona, Tucson, AZ 85721, USA; msteinru@lpl.arizona.edu \\ ${ }^{2}$ Department of Physics, University of Oxford, Oxford, OX1 3PU, UK \\ ${ }^{3}$ BAER Institute/NASA Ames Research Center, Moffett Field, CA 94043, USA \\ Received 2018 August 6; revised 2019 May 15; accepted 2019 May 16; published 2019 July 18
}

\begin{abstract}
On hot Jupiter exoplanets, strong horizontal and vertical winds should homogenize the abundances of the important absorbers $\mathrm{CH}_{4}$ and $\mathrm{CO}$ much faster than chemical reactions restore chemical equilibrium. This effect, typically neglected in general circulation models (GCMs), has been suggested to explain discrepancies between observed infrared light curves and those predicted by GCMs. On the nightsides of several hot Jupiters, GCMs predict outgoing fluxes that are too large, especially in the Spitzer $4.5 \mu \mathrm{m}$ band. We modified the SPARC/MITgcm to include disequilibrium abundances of $\mathrm{CH}_{4}, \mathrm{CO}$, and $\mathrm{H}_{2} \mathrm{O}$ by assuming that the $\mathrm{CH}_{4} / \mathrm{CO}$ ratio is constant throughout the simulation domain. We ran simulations of hot Jupiter HD $189733 \mathrm{~b}$ with eight $\mathrm{CH}_{4} / \mathrm{CO}$ ratios. In the more likely COdominated regime, we find temperature changes $\geqslant 50-100 \mathrm{~K}$ compared to the simulation for equilibrium chemistry across large regions. This effect is large enough to affect predicted emission spectra and should thus be included in GCMs of hot Jupiters with equilibrium temperatures between 600 and $1300 \mathrm{~K}$. We find that spectra in regions with strong methane absorption, including the Spitzer 3.6 and $8 \mu \mathrm{m}$ bands, are strongly impacted by disequilibrium abundances. We expect chemical quenching to result in much larger nightside fluxes in the $3.6 \mu \mathrm{m}$ band, in stark contrast to observations. Meanwhile, we find almost no effect on predicted observations in the $4.5 \mu \mathrm{m}$ band, because the changes in opacity due to $\mathrm{CO}$ and $\mathrm{H}_{2} \mathrm{O}$ offset each other. We thus conclude that disequilibrium carbon chemistry cannot explain the observed low nightside fluxes in the $4.5 \mu \mathrm{m}$ band.
\end{abstract}

Key words: hydrodynamics - methods: numerical - planets and satellites: atmospheres - planets and satellites: gaseous planets - planets and satellites: individual (HD 189733b) - radiative transfer

Supporting material: data behind figures

\section{Introduction}

Close-in extrasolar giant planets, known as hot Jupiters, are the best characterized exoplanets to date. Due to their proximity to their host stars, they are expected to be tidally locked. This creates strong temperature contrasts between the permanent dayside and the nightside. For the temperature ranges of typical hot Jupiters, assuming chemical equilibrium, these temperature contrasts translate to large horizontal gradients in the abundances of methane $\left(\mathrm{CH}_{4}\right)$ and carbon monoxide $(\mathrm{CO})$, two important infrared absorbers in the atmospheres of hot Jupiters. Carbon is preferentially found in $\mathrm{CH}_{4}$ at high pressures and low temperatures, while, $\mathrm{CO}$ is the dominant carbon-bearing species at low pressures and high temperatures. Most models of hot Jupiters, especially three-dimensional general circulation models (GCMs) that allow for realistic representation of opacities and the effect that chemical composition exerts on them, assume equilibrium chemistry as a basis for calculating opacities. However, the stark day-night temperature contrast also drives a vigorous atmospheric circulation, with a strong eastward equatorial jet advecting thermal energy from the dayside to the nightside and strong vertical mixing (e.g., Showman \& Guillot 2002; Dobbs-Dixon \& Lin 2008; Heng et al. 2011; Showman \& Polvani 2011; Thrastarson \& Cho 2011; Perna et al. 2012; Rauscher \& Menou 2012; Dobbs-Dixon \& Agol 2013; Parmentier et al. 2013; Mayne et al. 2014; Kataria et al. 2016; Mendonça et al. 2016). In addition, at low pressures ( $\lesssim 1$ bar), the chemical timescale on which the interconversion between $\mathrm{CH}_{4}$ and $\mathrm{CO}$ acts, becomes very long. Therefore, an air parcel is advected much faster than its $\mathrm{CH}_{4}$ and $\mathrm{CO}$ abundances can adapt to the local equilibrium values. This process, called quenching, is expected to vertically and horizontally homogenize the abundances of $\mathrm{CH}_{4}$ and $\mathrm{CO}$ (as well as of many other species, including $\mathrm{N}_{2}$ and $\mathrm{NH}_{3}$ ) in the near-infrared photospheres (located roughly between 1 bar and 1 mbar) of hot Jupiters (Cooper \& Showman 2006; Agúndez et al. 2012, 2014; Drummond et al. 2018a, 2018b).

These disequilibrium abundances can have a significant effect on the opacities and thus the radiative transfer. Including this effect in GCMs could potentially affect the thermal structure and atmospheric circulation, as well as the predicted spectra and phase curves. In fact, this has been proposed as a solution for the observed discrepancy between phase curves predicted by state-of-the-art GCMs that assume equilibrium chemistry and observations with the Spitzer Space Telescope (Knutson et al. 2012). GCMs overpredict the nightside fluxes in the Spitzer $4.5 \mu \mathrm{m}$ band for the hot Jupiters HD 189733b (Knutson et al. 2012), HD 209458b (Zellem et al. 2014), and WASP-43b (Stevenson et al. 2017). Within this wavelength bandpass, $\mathrm{CO}$ has a strong absorption band. For relatively cool hot Jupiters, such as HD 189733b, transport-induced disequilibrium chemistry is expected to enhance the $\mathrm{CO}$ abundance on the nightside compared to the equilibrium chemistry value (Cooper \& Showman 2006; Agúndez et al. 2014). Knutson et al. (2012) argued that this would lead to increased opacity in the $4.5 \mu \mathrm{m}$ band, such that the outgoing radiation in that band would probe higher, cooler regions of the atmosphere, decreasing the flux emitted in this band. In addition to 
changing the pressure level from which the outgoing radiation is emitted, the altered opacities can also affect the thermal structure and the atmospheric circulation in a GCM, an effect not considered in the argumentation of Knutson et al. (2012). So far, this effect has only been taken into account by Drummond et al. (2018a, 2018b).

The goal of this study is to better quantify how the combination of these two effects of disequilibrium carbon chemistry (the change in the level from which radiation escapes to space and the change in thermal structure) affects the thermal emission spectra predicted from GCMs. We assume that the abundance ratio of $\mathrm{CH}_{4}$ to $\mathrm{CO}$ is constant throughout the entire simulation domain and treat it as a free parameter. This simple approach allows us to explore a broader parameter range than Drummond et al. (2018a, 2018b) and to focus on the radiative effects. Our approach is justified by the findings of previous studies. Coupling a simple chemical relaxation scheme to a GCM of HD 209458b, both Cooper \& Showman (2006) and Drummond et al. (2018b) found that the $\mathrm{CO}$ and $\mathrm{CH}_{4}$ abundances are homogenized everywhere above the $\sim 3$ bar level. In a later study (published while this paper was in the peer-review process), Drummond et al. (2018a) find that the same is true for HD 189733b. Agúndez et al. (2014) instead used a full kinetic network in a pseudo-2D framework that was able to capture vertical and horizontal transport, and included photochemistry on the dayside. They found that quenching homogenized the $\mathrm{CO}$ and $\mathrm{CH}_{4}$ abundances at pressures between $\sim 1$ and $\sim 10^{-4}$ bars on HD 189733b. (At lower pressures, photochemical processes destroy $\mathrm{CH}_{4}$ on the dayside.) While these studies disagree on the relative importance of vertical versus horizontal quenching, all of them conclude that the abundances of $\mathrm{CO}$ and $\mathrm{CH}_{4}$ should be homogeneous in the near-infrared photosphere, justifying our assumption.

However, coupling a chemical relaxation scheme to their GCM, Mendonça et al. (2018b) found that on WASP-43b, the $\mathrm{CH}_{4}$ abundances were only homogenized horizontally but not vertically. A similar behavior is seen in the case of HD 209458b by Agúndez et al. (2014) (though only for their nominal eddy diffusion profile). While there are several differences in the models and planet parameters used that could contribute to this different outcome, perhaps the most important factors are the hotter dayside and the weak vertical mixing in both of these models. If horizontal transport dominates over vertical transport in setting the disequilibrium abundances, as these two papers find, then whether abundances are homogenized only horizontally or vertically and horizontally depends on whether vertical quenching happens in the hottest region of the dayside. Abundances thus are homogenized vertically and horizontally if in the hottest regions of the dayside the vertical mixing timescale is shorter than the chemical timescale. If vertical mixing is very weak or the dayside is too hot (leading to a shorter chemical timescale), this condition is not fulfilled and abundances are only homogenized horizontally and not vertically. In the case of Agúndez et al. (2014), the hotter dayside compared to Drummond et al. (2018b) at low pressures is largely due to their assumption of a thermal inversion on the dayside of HD 209458b. Mendonça et al. (2018b) look at WASP-43b, for which Kataria et al. (2015) also found a larger day-night contrast and hotter dayside compared to HD 209458 b due to its shorter orbital period and larger gravity. Our study looks at HD 189733b, which has a significantly lower zero-albedo equilibrium temperature than HD 209458b and WASP-43b. Since the chemical timescale dramatically increases with decreasing temperature, it is likely that abundances are homogenized horizontally and vertically on this planet, and both published studies looking at HD 189733b confirm this (Agúndez et al. 2014; Drummond et al. 2018a).

With the focus of these previous studies being on chemistry, most of them did not self-consistently calculate the radiative transfer, and thus were not able to quantify the effect of the changed opacities on the temperature structure in their models. In their GCM, Cooper \& Showman (2006) used a Newtonian cooling scheme, in which the temperature relaxes toward a prescribed temperature profile at each point in the atmosphere. Agúndez et al. (2014) used a prescribed background pressuretemperature profile, like typical kinetic networks (in this case derived from a GCM assuming equilibrium chemistry). Mendonça et al. (2018b) use a double-gray radiative transfer scheme. Only Drummond et al. (2018a, 2018b), whose GCM uses state-of-the-art radiative transfer with correlated $k$ coefficients, included the effect of the changed opacities on the temperature structure in a GCM. Our approach complements previous studies by focusing on the radiative transfer rather than on chemistry. The photospheric disequilibrium $\mathrm{CH}_{4}$ and $\mathrm{CO}$ abundances found in coupled chemistry-circulation models such as that of Drummond et al. (2018b) strongly depend on the temperature profile in the transition region between equilibrium chemistry and disequilibrium chemistry ( $\sim 1$ to 10 bars) (Moses et al. 2011; Venot et al. 2014). However, the temperature profile in this region depends on the initial temperature profile used in the GCM (Amundsen et al. 2016), assumptions about the interior heat flux (e.g., Guillot \& Showman 2002; Burrows et al. 2003; Fortney et al. 2008), and dissipation in deep layers (e.g., Guillot \& Showman 2002; Komacek \& Youdin 2017; Tremblin et al. 2017). Furthermore, typical GCM simulations only resolve dynamic mixing through the large-scale circulation. If unresolved sub-grid-scale turbulence is relevant near the quench level (e.g., Menou 2019), it might further alter the quenched abundances. Uncertainties in reaction rates can also affect the resulting abundances (e.g., Visscher \& Moses 2011). Overall, although it is well established that the $\mathrm{CH}_{4} / \mathrm{CO}$ ratio should be reasonably homogenized throughout the photosphere, many unconstrained factors will determine the actual value of this ratio. Studying how the thermal response and the resulting emission spectra and phase curves depend on the $\mathrm{CH}_{4} / \mathrm{CO}$ ratio, as is our goal, thus adds to the overall understanding of the effects of disequilibrium chemistry.

We choose to focus on HD 189733b. It has a lower temperature than HD 209458b and WASP-43b, so disequilibrium effects are expected to be stronger on this planet. The latter two planets are hot enough that in simulations assuming equilibrium chemistry, $\mathrm{CO}$ is the dominant carbon species at all longitudes and latitudes (Showman et al. 2009; Drummond et al. 2018b). In contrast, on the cooler HD 189733b, one expects that in chemical equilibrium the dayside is dominated by $\mathrm{CO}$, while the nightside is dominated by $\mathrm{CH}_{4}$ (see Figure 1). HD 189733b thus occupies an interesting point in the parameter space: regardless of the quenched $\mathrm{CH}_{4}$ and $\mathrm{CO}$ abundances, including disequilibrium chemistry will change which of the two species is dominant on about half of the planet. The radiative effects of disequilibrium chemistry are thus expected to play a larger role than on hotter planets. In 

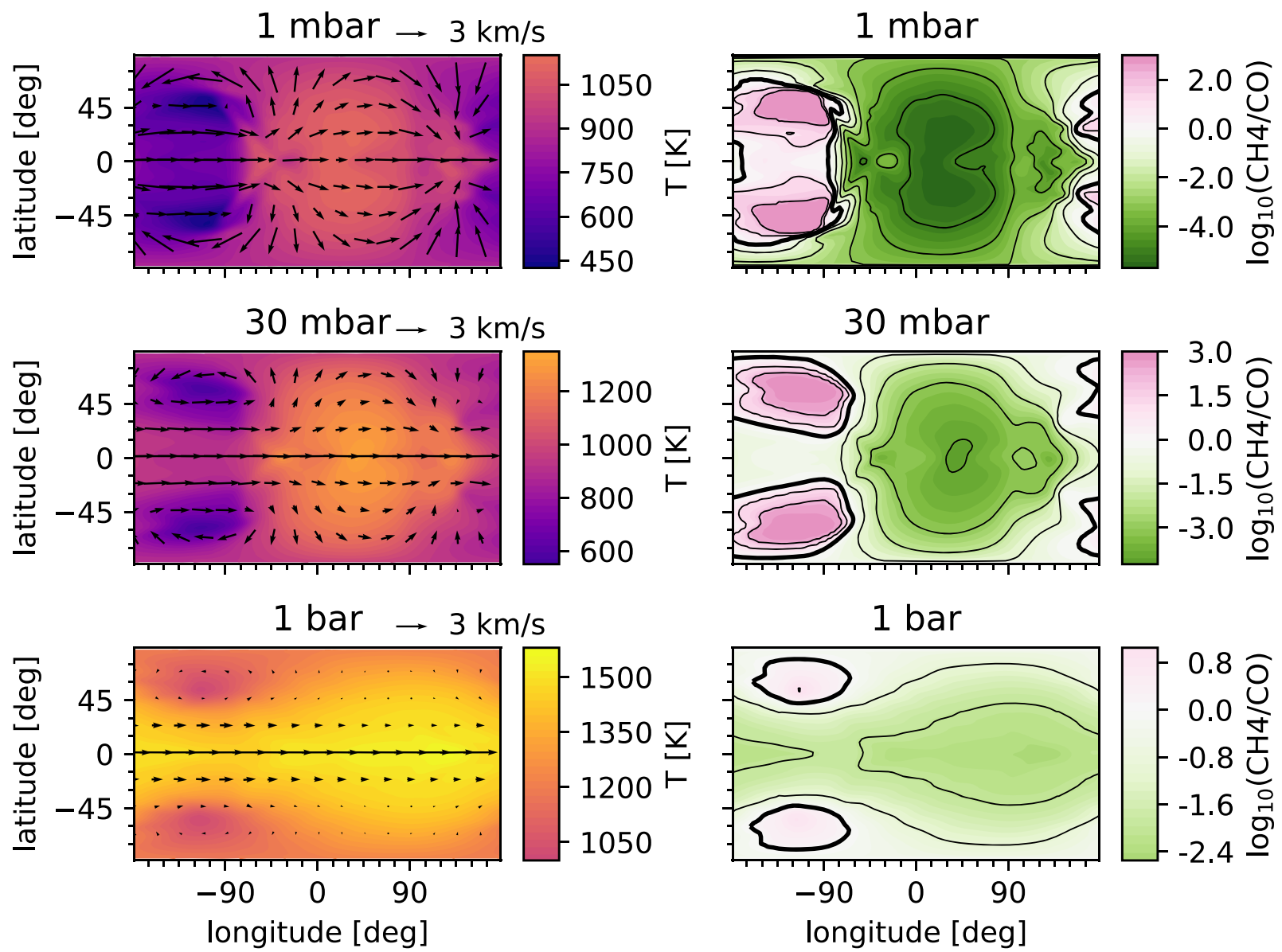

Figure 1. Left: horizontal temperature maps of the reference simulation (equilibrium chemistry) at pressures of $1 \mathrm{mbar}, 30 \mathrm{mbar}$, and 1 bar (top to bottom). The substellar point $\left(0^{\circ}\right.$ longitude, $0^{\circ}$ latitude $)$ is at the center of each panel. The arrows represent the velocities of the horizontal component of the wind. Right: horizontal maps of the abundance ratio of methane to carbon monoxide assuming chemical equilibrium. Regions dominated by methane are magenta while those dominated by carbon monoxide are green. The contours are evenly spaced in $\log$ space and mark $\log _{10}\left(\mathrm{CH}_{4} / \mathrm{CO}\right)=(-4,-3,-2,-1,0,1,2)$. The zero contour (corresponding to $\left.\mathrm{CH}_{4} / \mathrm{CO}=1\right)$ is thickened.

addition, unlike for HD 209458b, there exist phase-curve observations for HD 189733b in multiple Spitzer bandpasses (Knutson et al. 2007, 2009, 2012; Agol et al. 2010), providing stronger observational constraints.

\section{Methods}

We use the SPARC/MITgcm model (Showman et al. 2009) to perform simulations of hot Jupiter HD 189733b. This model couples the two-stream, non-gray radiative transfer code of Marley \& McKay (1999) to the MITgcm GCM of Adcroft et al. (2004) and has previously been applied to a wide range of hot Jupiters and other exoplanets (e.g., Showman et al. 2009, 2013, 2015; Lewis et al. 2010, 2014; Kataria et al. 2013, 2014, 2015; Parmentier et al. 2013, 2018).

\subsection{Dynamics}

Using the MITgcm (Adcroft et al. 2004), we solve the threedimensional global primitive equations on a cubed sphere grid. The primitive equations are valid for stably stratified shallow atmospheres. Horizontal noise is smoothed with a fourth-order Shapiro filter (Shapiro 1970).

The key model parameters are summarized in Table 1 . We assume a gravity of $g=22.86 \mathrm{~m} \mathrm{~s}^{-2}$, a planetary radius of $7.9559 \times 10^{7} \mathrm{~m}$ and a rotation period equal to the orbital period of 2.22 days, because the planet is assumed to be in
Table 1

Model Parameters

\begin{tabular}{lrl}
\hline \hline Parameter & Value & Units \\
\hline Radius & $7.9559 \times 10^{7}$ & $\mathrm{~m}$ \\
Gravity & 22.86 & $\mathrm{~m} \mathrm{~s}^{-2}$ \\
Rotation period & 2.22 & $\mathrm{days}$ \\
Semimajor axis & 0.03142 & $\mathrm{au}$ \\
Specific heat capacity & $1.3 \times 10^{4}$ & $\mathrm{~J} \mathrm{~kg}^{-1} \mathrm{~K}^{-1}$ \\
Specific gas constant & 3714 & $\mathrm{~J} \mathrm{~kg}^{-1} \mathrm{~K}^{-1}$ \\
Interior flux & 0 & $\mathrm{~W} \mathrm{~m}^{-2}$ \\
Horizontal resolution & $\mathrm{C} 32^{\mathrm{a}}$ & \\
Vertical resolution & 53 & layers \\
Lower pressure boundary & $2 \times 10^{-6}$ & $\mathrm{bar}$ \\
Upper pressure boundary & 200 & $\mathrm{bar}$ \\
Hydrodynamic time step & 25 & $\mathrm{~s}$ \\
Radiative time step & 50 & $\mathrm{~s}$ \\
\hline
\end{tabular}

Note.

${ }^{\text {a }}$ Equivalent to a resolution of $128 \times 64$ on a longitude-latitude grid.

synchronous rotation. We use a value of $c_{p}=1.3 \times 10^{4} \mathrm{~J} \mathrm{~kg}^{-1}$ $\mathrm{K}^{-1}$ for the specific heat capacity and $\kappa=R / c_{p}=2 / 7$, where $R$ is the specific gas constant. These values are appropriate for hydrogen-dominated atmospheres. Our simulations use a horizontal resolution of $\mathrm{C} 32$, which is roughly equivalent to a resolution of $128 \times 64$ on a longitude-latitude grid. The 
pressure spans from 200 bars to $2 \mu$ bar and is resolved by 53 vertical levels. We use a time step of $25 \mathrm{~s}$, run the simulations for 1000 days, and then average over the last 100 days. Convergence tests in previous studies using the SPARC/ MITgcm have shown that simulations have sufficiently converged at that point. Specifically, the emitted infrared flux changes by less than a few per cent between integration times of $\approx 1000$ and $\approx 4000$ days (Showman et al. 2009, Figure 12).

\subsection{Radiative Transfer}

The radiative transfer code in our model is based on the plane-parallel code of Marley \& McKay (1999). This code was originally developed to study Titan's atmosphere (McKay et al. 1989) and has since been applied to Uranus (Marley \& McKay 1999), brown dwarfs (Marley et al. 1996, 2002; Burrows et al. 1997), and hot Jupiters (Fortney et al. 2005, 2008; Showman et al. 2009). The code uses the deltadiscrete ordinate method (Toon et al. 1989) for the incident stellar flux, while the thermal flux is calculated using the twostream source function method (also of Toon et al. 1989). Molecular opacities are treated using the correlated- $k$ method (e.g., Goody \& Yung 1989). In each frequency bin, the opacity information from line-by-line calculations using as many as $10,000-100,000$ frequency bins is turned into a cumulative distribution of opacities, which is then described by eight $k$-coefficients. We use 11 frequency bins spanning from 0.26 to $325 \mu \mathrm{m}$ (see Kataria et al. 2013). The correlated- $k$ method is the most sophisticated and accurate treatment of opacities in GCMs of exoplanets to date and is used both in the SPARC/MITgcm and in the adaptation for hot Jupiter of the UK Met Office model (Amundsen et al. 2016).

In order to combine the opacities from different species within the atmosphere, we employ the approach of pre-mixed opacities, meaning that the $k$-coefficients of the mixture are derived from line-by-line opacities that are calculated for a mixture with chemical abundances that are specified functions of temperature and pressure (typically derived from equilibrium chemistry). This approach is fast and accurate. We use the equilibrium chemistry abundances for solar metallicity of Lodders \& Fegley (2002) and Visscher et al. (2006) with the modifications for $\mathrm{CH}_{4}, \mathrm{CO}$, and $\mathrm{H}_{2} \mathrm{O}$ described in Section 2.3 and the opacities of Freedman et al. (2008) including the updates of Freedman et al. (2014). Note that the updated opacities result in changes in the thermal structure and the resulting equilibrium chemistry phase curve compared to Showman et al. (2009) and Knutson et al. (2012) (see Section 4.2). The disadvantage of this method is that it leaves little flexibility for varying abundances due to disequilibrium chemistry. However, alternative methods combining the $k$-coefficients of individual species on the fly come at a much higher computational cost and are thus impractical when coupled to GCMs (as discussed in Amundsen et al. 2017). To explore the effect of disequilibrium abundances, we thus take advantage of the fact that due to strong horizontal and vertical mixing, the disequilibrium abundances of $\mathrm{CH}_{4}$ and $\mathrm{CO}$ are expected to be close to constant throughout the photosphere, as further detailed in Section 2.3.

To obtain spectra and phase curves, we postprocess our simulation output using the same plane-parallel two-stream radiative transfer code as used in our full 3D simulations, but adopting 196 wavelength bins instead of 11 to yield improved spectral resolution. Given the time-averaged temperature structure from the GCM at a particular time, we calculate the outgoing flux in the line of sight to the observer for each atmospheric column. The fluxes are then combined into a weighted average across the disk to give the total flux received at a particular observing vantage point and at a particular time during the orbit. We do this calculation at many orbital phases (throughout which the Earth-facing hemisphere shifts in longitude) to assemble full-orbit phase curves at wavelengths of interest. This method is similar to the method described in Fortney et al. (2006), Showman et al. (2009), and Parmentier et al. (2016) and naturally takes into account limb darkening of the planet. We use a NextGen spectrum (Hauschildt et al. 1999) for HD 189733, a stellar radius of $0.805 R_{\odot}$ (Boyajian et al. $2015)$, and a planet-to-star radius ratio $R_{p} / R_{*}=0.0145$.

\subsection{Disequilibrium Chemistry}

All previous studies of hot Jupiters using the SPARC/ MITgcm utilized $k$-coefficient tables calculated assuming equilibrium chemistry. To explore the effect of transportinduced disequilibrium carbon chemistry, we instead assume in this study that the $\mathrm{CH}_{4}$ to $\mathrm{CO}$ ratio is quenched to a constant value throughout the entire atmosphere. This assumption is a good approximation at pressures lower than roughly 1 to 10 bars, as shown by Cooper \& Showman (2006), Agúndez et al. (2014), and Drummond et al. (2018a, 2018b). At higher pressures, the chemical timescale becomes shorter than the mixing timescale and the approximation of a constant quenched value breaks down. Since this study focuses on the effect on the outgoing radiation in the near-infrared, which probes pressures between 1 mbar and 1 bar (see Figure 9 in Showman et al. 2009 and Figures 12 and 14 in Moses et al. 2011), we believe this approximation is justified. The deep regions of the atmosphere (deeper than 10 bars) also have extremely small net fluxes compared to the fluxes in the observable atmosphere (where the incoming starlight is absorbed and radiation is escaping to space), and so the dynamics in the observable atmosphere are not strongly sensitive to modest changes in the opacities and fluxes in the deep ( $p>10$ bar) atmosphere. In addition, because of the low net fluxes, the error in opacity we make by extending constant $\mathrm{CH}_{4} / \mathrm{CO}$ ratios to the deep atmosphere is expected to have a relatively small effect on the temperature structure. We expect the error in the temperature profile in the deep atmosphere to be smaller than, or at most comparable to, the uncertainty due to the unknown internal heat flux of hot Jupiters and certainly smaller than the error due to the limited integration time. (Because of the low net fluxes and long radiative timescales in the deep atmosphere, the time it takes for 3D GCM simulations to converge in the deep atmosphere far exceeds simulation runtimes feasible with state-of-the-art computational facilities. This problem is not unique to our model, but universal to 3D GCMs of hot Jupiters when realistic radiative transfer is included.)

Our approximation of a constant $\mathrm{CH}_{4} / \mathrm{CO}$ ratio also breaks down at pressures lower than $\sim 10^{-4}$ bars, because on the dayside $\mathrm{CH}_{4}$ is destroyed photochemically in these regions (Moses et al. 2011; Agúndez et al. 2014). Again, this process is not expected to affect the broadband emission spectra that we are interested in or the temperatures in the regions probed by broadband emission.

The quenched $\mathrm{CH}_{4} / \mathrm{CO}$ ratio can in general be constrained by comparing the chemical timescale and the mixing timescale. In a $1 \mathrm{D}$ picture, the quenched abundances are approximately 
given by the abundances at the point at which the two timescales are equal. In practice, however, the vertical mixing timescale in 1D and 2D models depends on the assumptions about the strength of vertical mixing (parameterized through the eddy diffusion coefficient $K_{z z}$ ), and the chemical timescale depends on the reaction rates, some of which are not well known in the range of pressure and temperature encountered in hot Jupiter atmospheres. A variety of different 1D and pseudo-2D thermochemical kinetics models of HD 189733b find $\mathrm{CH}_{4} / \mathrm{CO}$ ratios roughly between 0.001 and 0.5 (Moses et al. 2011; Visscher \& Moses 2011; Agúndez et al. 2014; Drummond et al. 2016). However, $\mathrm{CH}_{4} / \mathrm{CO}$ ratios larger than one might also be possible if vertical mixing is very strong (Tsai et al. 2018). GCM simulations with simplified chemical schemes have focused on the hotter HD 209458b and find $\mathrm{CH}_{4} / \mathrm{CO}$ ratios $\sim 0.01$ (Cooper \& Showman 2006; Drummond et al. 2018b). For HD 189733b, Drummond et al. (2018a) find $\mathrm{CH}_{4} / \mathrm{CO}$ ratios between 0.1 and 0.2 . We thus choose to treat the $\mathrm{CH}_{4} / \mathrm{CO}$ ratio as a free parameter and perform eight simulations varying this ratio from 0.001 to 100 as well as a reference simulation assuming equilibrium chemistry. Although kinetics models favor $\mathrm{CH}_{4} / \mathrm{CO}$ ratios $<1$, we include the full range for completeness.

\subsubsection{Water and $\mathrm{CO}_{2}$ Abundances}

The net reaction that converts $\mathrm{CH}_{4}$ to $\mathrm{CO}$ and vice versa is

$$
\mathrm{CH}_{4}+\mathrm{H}_{2} \mathrm{O} \rightleftharpoons \mathrm{CO}+3 \mathrm{H}_{2} \text {. }
$$

Together with the $\mathrm{CH}_{4}$ and $\mathrm{CO}$ abundances, the water abundance thus changes as well. $\mathrm{CO}$ and $\mathrm{H}_{2} \mathrm{O}$ are the only major oxygen-bearing species in the region where quenching dominates the abundances (Moses et al. 2011), and the total number of oxygen atoms has to be preserved. As a consequence, when reaction (1) is quenched, the water abundance is frozen at a constant value as well. This value is directly tied to the $\mathrm{CH}_{4}$ and $\mathrm{CO}$ abundances (e.g., Moses et al. 2011, Figures 4, 5, and 8). As long as either $\mathrm{CO}$ or $\mathrm{CH}_{4}$ dominates over the other, a change in the $\mathrm{CH}_{4} / \mathrm{CO}$ ratio results in only a marginal change in the water abundance. However, when transitioning from the CO-dominated to the $\mathrm{CH}_{4}$-dominated regime, the water abundance varies by a factor of $\approx 2$. Assuming equilibrium chemistry, the water abundance thus varies by this factor of $\approx 2$ between the dayside and nightside of HD 189733b, as can be seen in Figure 2. Including horizontal and vertical transport, however, the water abundance is expected to remain constant between dayside and nightside due to quenching (Agúndez et al. 2014, Figure 12). Since water is such an important infrared absorber, it is necessary to adjust the water abundance in our model to reflect this. We achieve this by adjusting the water abundance such that the total number of oxygen atoms present in water and $\mathrm{CO}$ is conserved. The $\mathrm{C} / \mathrm{H}$ and $\mathrm{O} / \mathrm{H}$ ratios thus remain at solar values, consistent with our general assumption of solar elemental abundances.

We leave the abundance of $\mathrm{CO}_{2}$ unchanged from its equilibrium chemistry value, because it is not the dominant carbon species in either equilibrium or disequilibrium chemistry and its deviations from equilibrium chemistry are expected to remain moderate compared to those of $\mathrm{CH}_{4}$ (Agúndez et al. 2014).

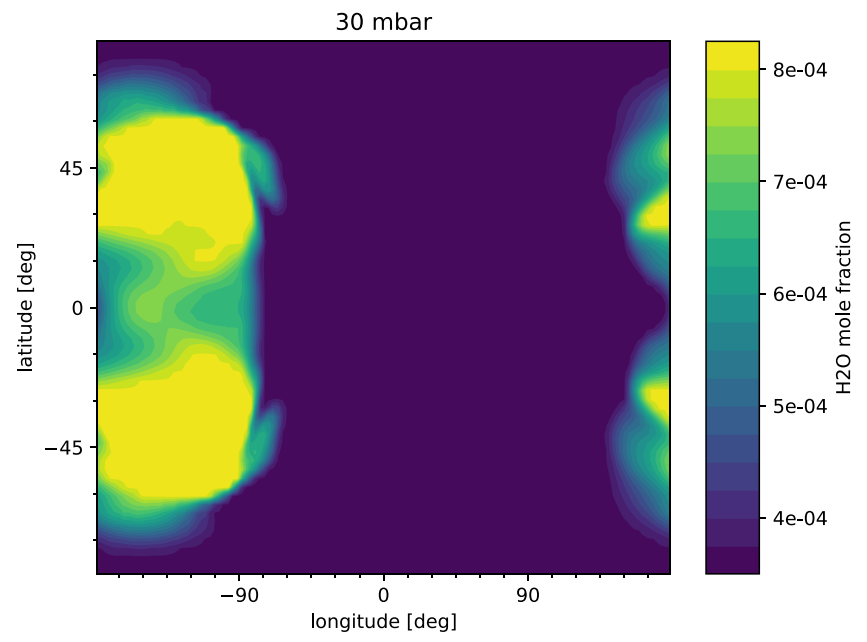

Figure 2. Water abundance at the $30 \mathrm{mbar}$ level in the equilibrium chemistry simulation. The substellar point is at the center of the panel. In disequilibrium chemistry, in contrast, the abundances are expected to be homogenized between the dayside and nightside of the planet.

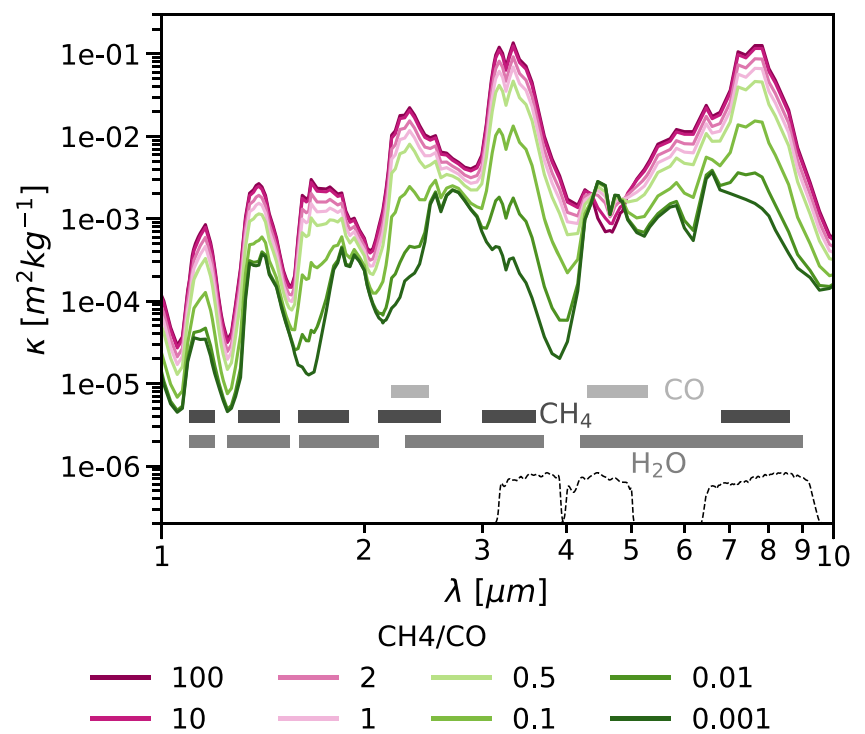

Figure 3. Opacities for different $\mathrm{CH}_{4} / \mathrm{CO}$ ratios at a temperature of $1000 \mathrm{~K}$ and a pressure of 30 mbar. Opacities for $\mathrm{CH}_{4}$-dominated ratios are plotted in magenta and those for $\mathrm{CO}$-dominated ratios in green. The locations of important absorption bands are shown as gray bars toward the bottom of the figure. The dashed black lines indicate the filter sensitivity profiles for the Spitzer 3.6, 4.5, and $8 \mu \mathrm{m}$ bands.

\subsubsection{Changes in Opacity}

To illustrate the changes in opacity, the resulting nearinfrared opacities for different $\mathrm{CH}_{4} / \mathrm{CO}$ ratios at a typical photospheric pressure and temperature are shown in Figure 3. The plotted opacities represent the bin-averaged opacity for each of the 196 wavelength bins used for the postprocessing averaged over the inverse of the opacity. This kind of average is a good way of representing the effects on the broadband emission. Note that the average is only used for illustration in the figure-in the radiative transfer in the GCM and for postprocessing we use the correlated- $k$ method instead (with 11 and 196 bins, respectively). It is obvious from Figure 3 that for a higher $\mathrm{CH}_{4} / \mathrm{CO}$ ratio, the opacity significantly increases for almost all wavelengths in the near- and mid-infrared. This is mainly because $\mathrm{CH}_{4}$ has many broad absorption bands in the 


\section{CO dominated}

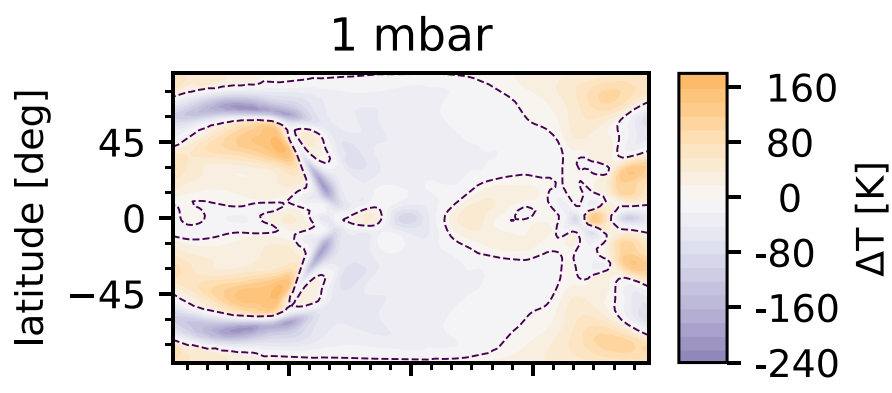

30 mbar

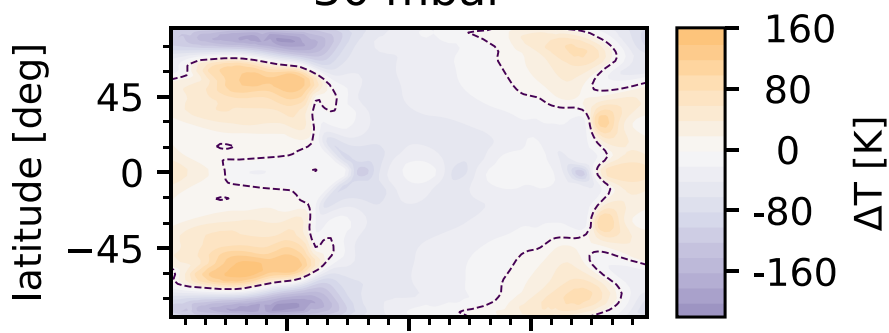

1 bar
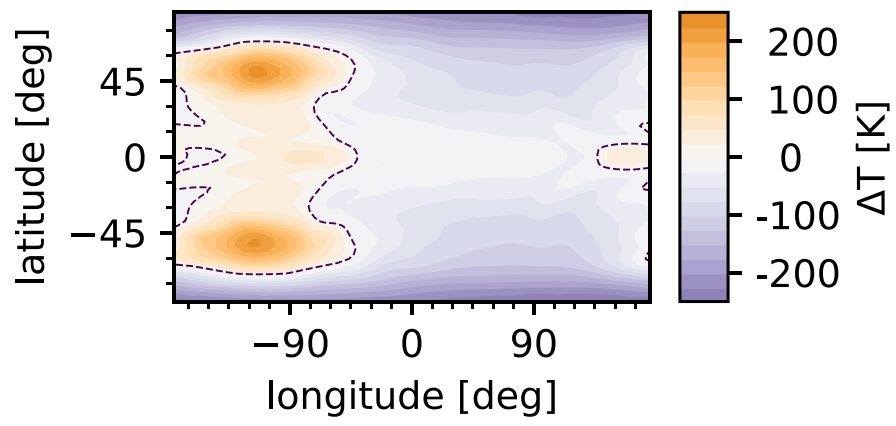

\section{CH4 dominated}

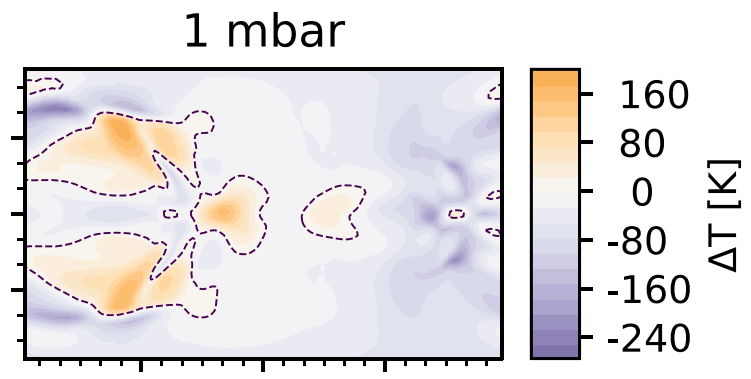

$30 \mathrm{mbar}$

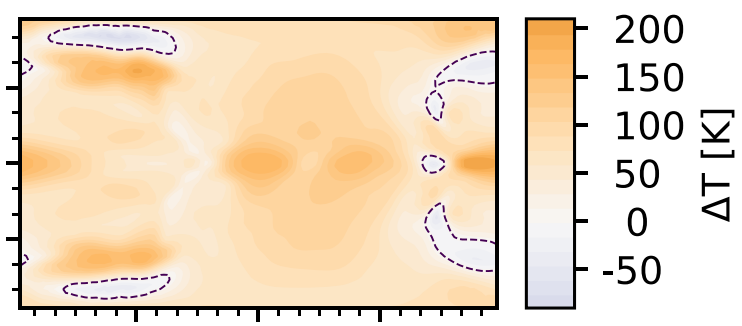

1 bar

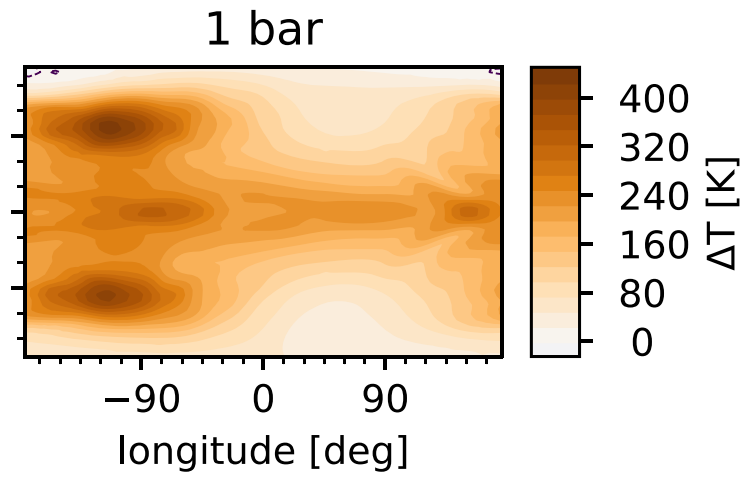

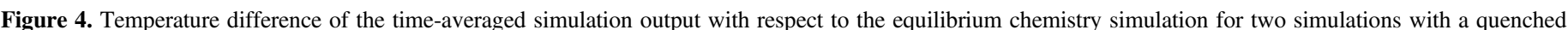

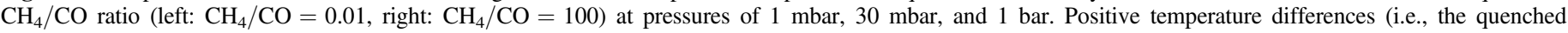
simulation is hotter) are shown in orange, negative ones in purple. Dashed lines indicate the zero contour.

near- and mid-IR while $\mathrm{CO}$ only has a few narrow absorption bands. On top of that, with increased $\mathrm{CH}_{4}$, the water abundance also increases by a factor of $\sim 2$. Similar to $\mathrm{CH}_{4}$, water has many broad absorption bands. The only spectral region in which the opacity decreases for high $\mathrm{CH}_{4} / \mathrm{CO}$ ratios is near the $\mathrm{CO}$ absorption band centered around $4.7 \mu \mathrm{m}$. Even in this region, the opacity decreases only by a factor of $\sim 2$, much less than it increases at most other wavelengths. This is likely because the water feature centered around $6.3 \mu \mathrm{m}$ contributes significantly to the total opacity in this region. Since a water molecule is added for every $\mathrm{CO}$ molecule that is removed, these two changes in opacity partially offset each other in this region.

\section{Results}

\subsection{Reference Simulation: Equilibrium Chemistry Case}

First, we give a brief overview of the reference simulation, which assumes equilibrium chemistry. The left column of Figure 1 shows the temperature and horizontal wind velocities at pressures of $1 \mathrm{mbar}, 30 \mathrm{mbar}$, and $1 \mathrm{bar}$. The pressure levels in the figure are chosen such that the lowest one shown (1 mbar, uppermost panel) is slightly above the photosphere at most infrared wavelengths, while the highest pressure level ( 1 bar, lowermost panel) is somewhat below the photosphere at most infrared wavelengths. The temperature and wind patterns are typical for a hot Jupiter: the simulation exhibits a large daynight temperature contrast, especially at low pressures, and a superrotating (eastward) equatorial jet. The hottest point is shifted east with respect to the substellar point. The coldest regions in the simulation are east of the antistellar point at roughly $40^{\circ}-50^{\circ}$ latitude, at the center of two large gyres.

At low pressures, where the radiative timescale is short compared to the dynamical timescale, the temperature difference between dayside and nightside is largest. In addition to the jet, there is a strong day-to-night flow. As pressure increases and the radiative timescale becomes longer, the eastward equatorial jet dominates more over the day-to-night flow. At a pressure of 1 bar, the equatorial jet efficiently transports heat from the dayside to the nightside, and longitudinal temperature differences along the equator are small. Winds at mid-latitudes are much smaller than at lower latitudes. The cold spots associated with the nightside gyres are still several hundred kelvin colder than the equatorial regions.

The ratio of the equilibrium chemistry abundances of $\mathrm{CH}_{4}$ and $\mathrm{CO}$ is plotted in the right column of Figure 1. In chemical 

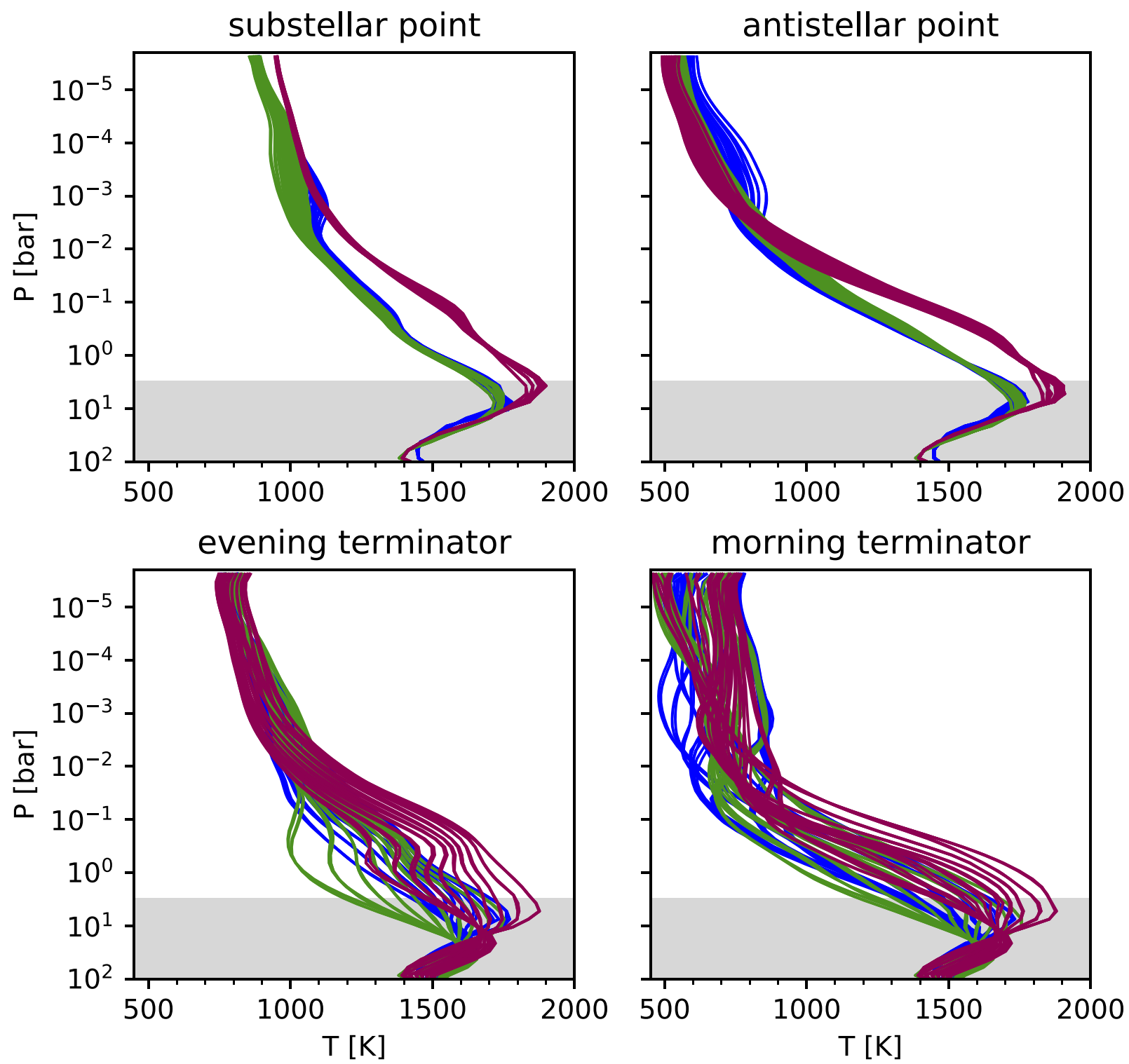

\section{equilibrium $\mathrm{CH} 4 / \mathrm{CO}=0.01=\mathrm{CH} 4 / \mathrm{CO}=100$}

Figure 5. Pressure-temperature profiles. The equilibrium chemistry simulation is shown in blue, the $\mathrm{CH}_{4} / \mathrm{CO}=0.01$ simulation in green, and the $\mathrm{CH}_{4} / \mathrm{CO}=100$ simulation in magenta. The shaded gray region indicates pressure ranges for which the simulation has not converged due to the long radiative timescale in deep layers of the atmosphere. Coincidentally, our assumption of a constant quenched $\mathrm{CH}_{4} / \mathrm{CO}$ ratio breaks down at a roughly similar pressure. Upper left: profiles within $10^{\circ}$ of the substellar point. Upper right: profiles within $10^{\circ}$ of the antistellar point. Lower left: profiles within $\pm 2^{\circ}$ of the evening terminator. Lower right: profiles within $\pm 2^{\circ}$ of the morning terminator. The temperature profiles in this figure are available as Data behind the Figure. The data used to create this figure are available.

equilibrium, $\mathrm{CH}_{4}$ is the dominant carbon species at high pressures and low temperatures, while carbon preferentially forms $\mathrm{CO}$ at low pressures and high temperatures. On isobars, abundances thus directly follow the temperature pattern. On the dayside, $\mathrm{CO}$ is the prevailing carbon species and is up to five orders of magnitude more abundant than $\mathrm{CH}_{4}$. At most locations of the nightside, the abundances of both species become more even, with $\mathrm{CH}_{4}$ prevailing. Methane abundances peak at the nightside cold spots at mid-latitudes. As with temperature gradients, the abundance gradients become much less pronounced deeper in the atmosphere: while the abundance ratios span over eight orders of magnitude at the $1 \mathrm{mbar}$ level, they differ by only three orders of magnitude at the 1 bar level.

\subsection{Simulations with Quenched Abundances: Thermal Structure}

In response to the different opacities, the thermal structure changes in the simulations with disequilibrium chemistry compared to the reference case. We consider two of the quenched simulations, $\mathrm{CH}_{4} / \mathrm{CO}=0.01$ and $\mathrm{CH}_{4} / \mathrm{CO}=100$, in detail to illustrate these changes for the CO-dominated and the $\mathrm{CH}_{4}$-dominated regimes. Figure 4 shows the temperature difference with respect to the reference case on isobars. Vertical pressure-temperature profiles from these two simulations along with the reference simulation are plotted in Figure 5.

In the CO-dominated regime (the regime favored by kinetics models), the dayside is cooler than in the equilibrium case, 

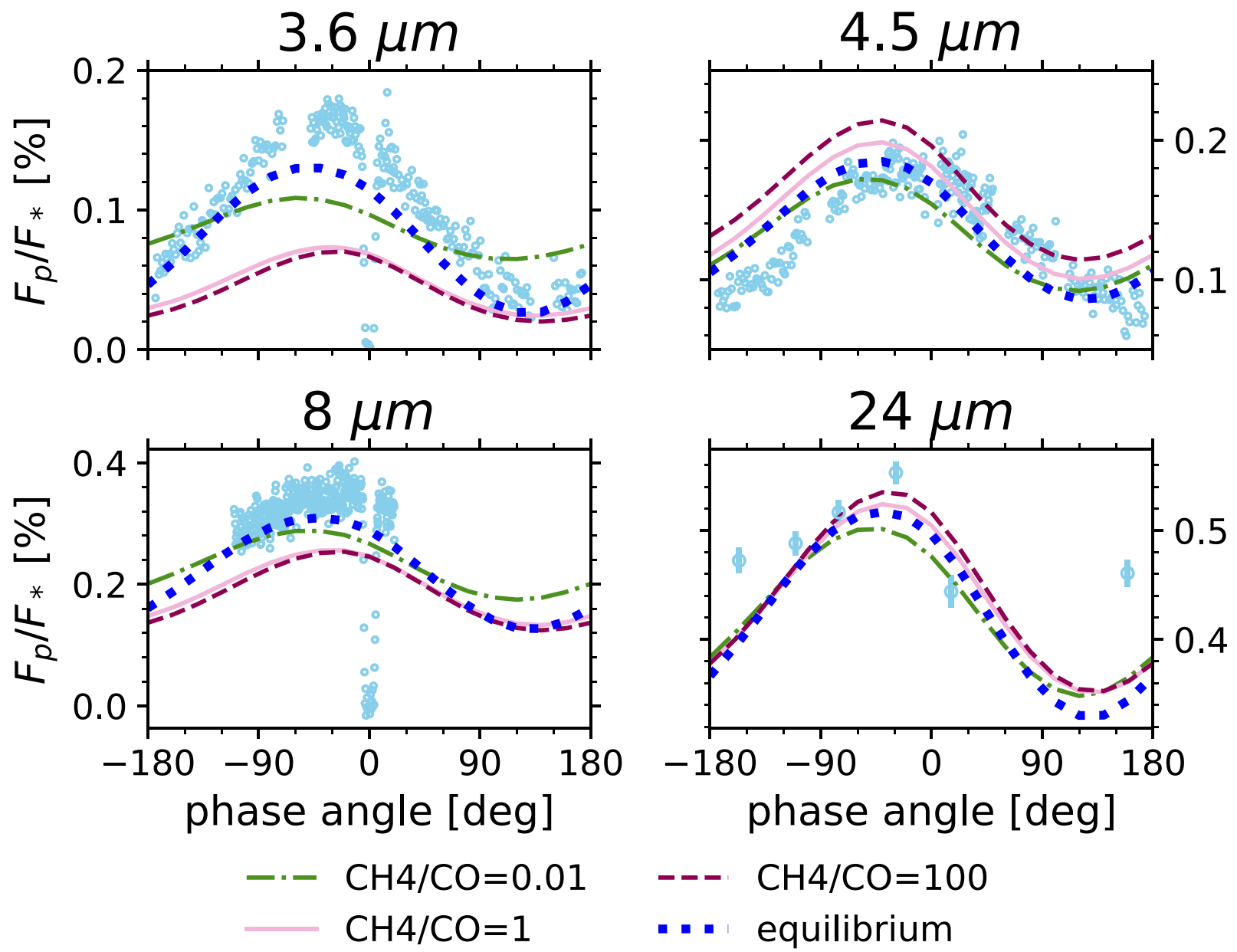

Figure 6. Phase curves predicted from our models and available observational data in the Spitzer 3.6, 4.5, 8, and $24 \mu \mathrm{m}$ bands. The green dashed-dotted line represents the $\mathrm{CH}_{4} / \mathrm{CO}=0.01$ simulation, the light pink solid line $\mathrm{CH}_{4} / \mathrm{CO}=1$, the magenta dashed line $\mathrm{CH}_{4} / \mathrm{CO}=100$, and the thick dotted blue line the reference simulation. The observational data (light blue circles) are the same as in Figure 12 in Knutson et al. (2012) and are taken from Knutson et al. (2012) (3.6 and $4.5 \mu \mathrm{m})$, Knutson et al. (2007, 2009), Agol et al. (2010) (8 $\mu \mathrm{m})$, and Knutson et al. (2009) (24 $\mu \mathrm{m})$. All model phase curves in this figure are available as Data behind the Figure. The data used to create this figure are available.

while the temperature of large parts of the nightside, including the mid-latitude cold spots, increases. Temperatures also drop at high latitudes, especially on the nightside. All of these effects can be seen in the left column of Figure 4. In the particular case shown $\left(\mathrm{CH}_{4} / \mathrm{CO}=0.01\right)$, dayside temperatures drop by about $50 \mathrm{~K}$ throughout most of the photosphere. This is also evident in the pressure-temperature profiles near the substellar point (upper left panel of Figure 4). A closer look at the pressuretemperature profiles reveals that the vertical temperature gradient is slightly larger than in the equilibrium chemistry case near the substellar point, but slightly smaller at the antistellar point. This is likely because on the dayside greenhouse gases $\left(\mathrm{CH}_{4}, \mathrm{H}_{2} \mathrm{O}\right)$ are added compared to the equilibrium composition while on the nightside greenhouse gases are taken away. The $\mathrm{CH}_{4} / \mathrm{CO}=0.001$ simulation (not shown) displays very similar changes in thermal structure, but with a somewhat larger amplitude.

Our interpretation for why the dayside is cooler is related to energy balance. The changes in opacity lead to only slight changes in albedo, so for all the $\mathrm{CH}_{4} / \mathrm{CO}$ ratios considered, the total amount of absorbed starlight is nearly the same. Since the simulations achieve an approximate energy balance (energy gained equals energy lost), this means that the total IR energy radiated to space (from the entire planet) is also nearly the same regardless of the $\mathrm{CH}_{4} / \mathrm{CO}$ ratio. Compared to equilibrium chemistry, the overall infrared opacity decreases on the nightside. Therefore, the radiation on the nightside escapes to space from deeper levels, where the atmosphere tends to be hotter, leading to a larger flux being radiated away. As a consequence, less energy is returned to the dayside and the dayside cools, which in turn leads to a dayside radiating less IR flux.

In the (less likely) $\mathrm{CH}_{4}$-dominated case, the changes in temperature are more striking: below roughly the $10 \mathrm{mbar}$ level, temperatures are significantly higher at almost all longitudes and latitudes. In the $\mathrm{CH}_{4} / \mathrm{CO}=100$ simulation, the temperature difference with respect to the reference simulation reaches $200-400 \mathrm{~K}$ at the 1 bar level in the equatorial region (see right column of Figure 4). In this simulation, the location of the nightside cold spots shifts to slightly higher latitudes. In Figure 4, this is visible in the center right panel as the light blue regions and in the upper right panel as the two small deep purple regions at $\approx \pm 60^{\circ}$ latitude in conjunction with two orange regions at lower latitudes.

At the 1 mbar level, temperatures are similar to or slightly lower than in the reference simulation. Looking at the 

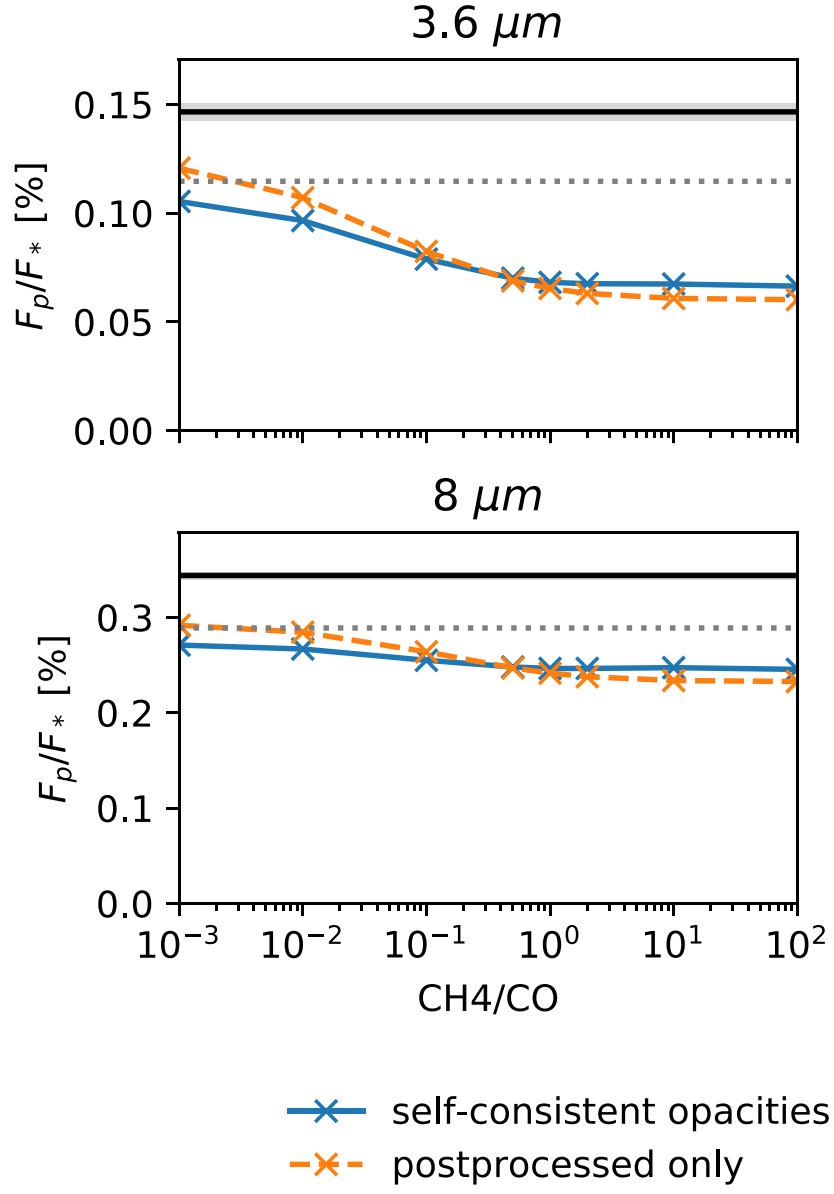

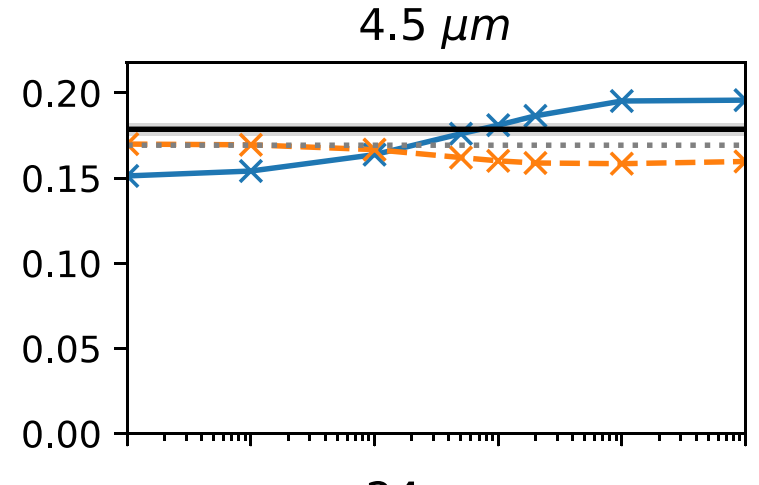

$24 \mu m$
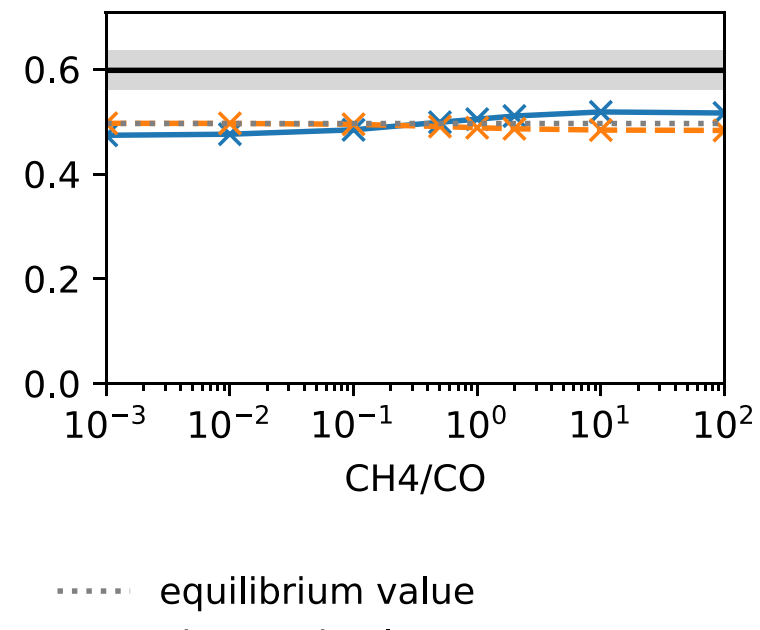

- observed value

Figure 7. Secondary eclipse depths for different quenched $\mathrm{CH}_{4} / \mathrm{CO}$ ratios. Values from the GCM simulations including the quenched opacities are shown as blue crosses connected by a solid line, while values obtained from postprocessing the equilibrium chemistry simulation are shown as orange crosses connected by a dashed line. The equilibrium value is indicated by the dashed gray line and the observed values from Knutson et al. (2012) are shown as a solid black line with the $1 \sigma$ errors shaded in gray.

temperature profiles in Figure 5, it is obvious that the temperature gradient between 1 mbar and 1 bar is steeper in the $\mathrm{CH}_{4}$-dominated case than for the other simulations at all four locations shown, signifying a stronger greenhouse effect. Given that in the $\mathrm{CH}_{4}$-dominated case the abundances of the greenhouse gases methane and water are enhanced compared to equilibrium chemistry by several orders of magnitude and a factor of $\sim 2$, respectively, at any location other than the nightside mid-latitude cold spots, this behavior is expected.

\subsection{Phase-curve Predictions and Comparison with Observations}

We postprocess the GCM results as described in Section 2.2 to obtain phase curves and emission spectra and compare them to the available observational data (Figure 6). In short, the fit with respect to the observational phase curve worsens substantially in the $3.6 \mu \mathrm{m}$ band for all quenched ratios while the changes in the $4.5 \mu \mathrm{m}$ band are negligible in the expected $\mathrm{CO}$-dominated case and lead to a worse fit in the $\mathrm{CH}_{4}$-dominated regime.

In the $3.6 \mu \mathrm{m}$ band, the phase-curve amplitude decreases for all quenched simulations compared to equilibrium chemistry. A similar, but less drastic behavior can be seen in the $8 \mu \mathrm{m}$ band. This behavior is expected for wavelength regions with strong methane absorption bands, including these two bandpasses. In equilibrium chemistry, the outgoing radiation on the dayside, where there is little methane, probes deeper, hotter layers while on the nightside, where more methane is present, the radiation is emitted from higher, cooler layers. Thus the temperature varies more strongly from day to night on the photosphere (which differs in pressure from day to night) in these bands than it does on isobars. This enhances the day-night contrast of the phase curve relative to what would occur if the photosphere were at constant pressure (see also Dobbs-Dixon \& Cowan 2017). When assuming a constant methane abundance throughout the atmosphere, however, regardless of the exact value, the outgoing radiation emerges from regions of similar pressure everywhere on the planet and the phase-curve amplitude decreases. Consistent with this picture, the dayside fluxes from the $\mathrm{CH}_{4} / \mathrm{CO}=0.01$ simulation are relatively close to those from the equilibrium chemistry simulation, while the nightside fluxes from the $\mathrm{CH}_{4}$-dominated simulations match those from equilibrium chemistry at the flux minimum.

In the $4.5 \mu \mathrm{m}$ band, the nightside fluxes are similar to or higher than the value from the equilibrium chemistry simulation for all quenched values. At first, this seems surprising, given that Knutson et al. (2012) base their argument for disequilibrium chemistry almost entirely on the fact that equilibrium chemistry models overpredict the $4.5 \mu \mathrm{m}$ nightside fluxes, arguing that adding $\mathrm{CO}$ on the nightside will shift the photosphere in this band to a higher, colder atmospheric layer 

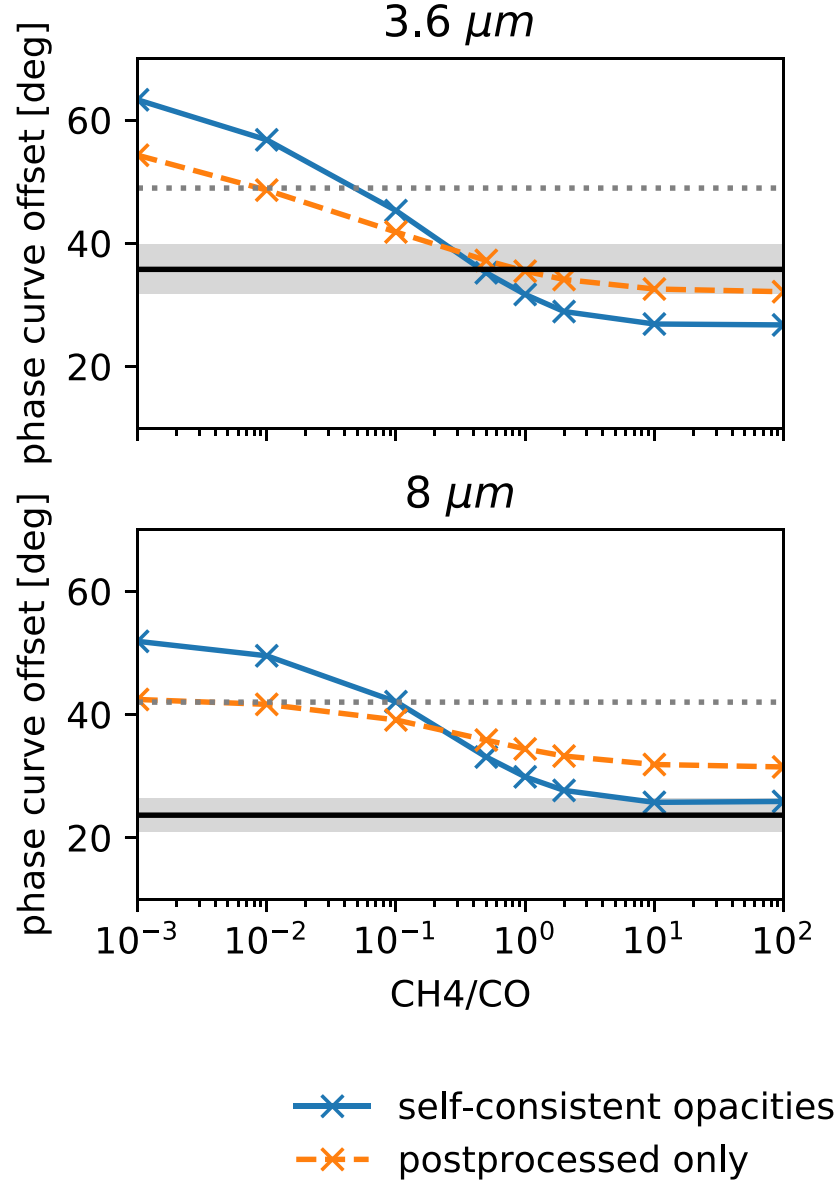

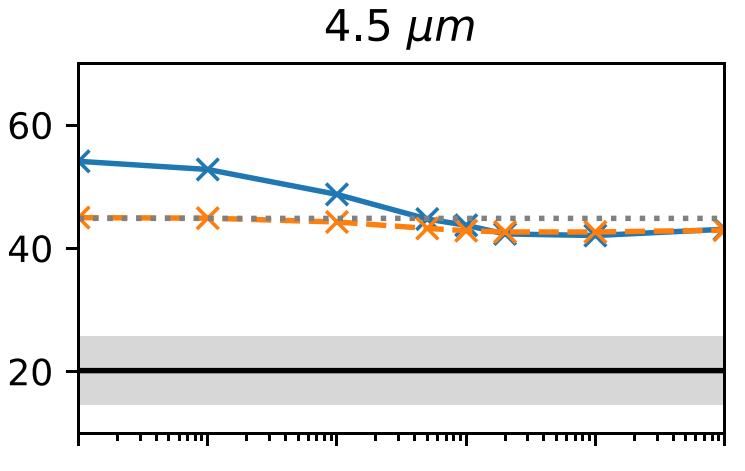

$24 \mu m$

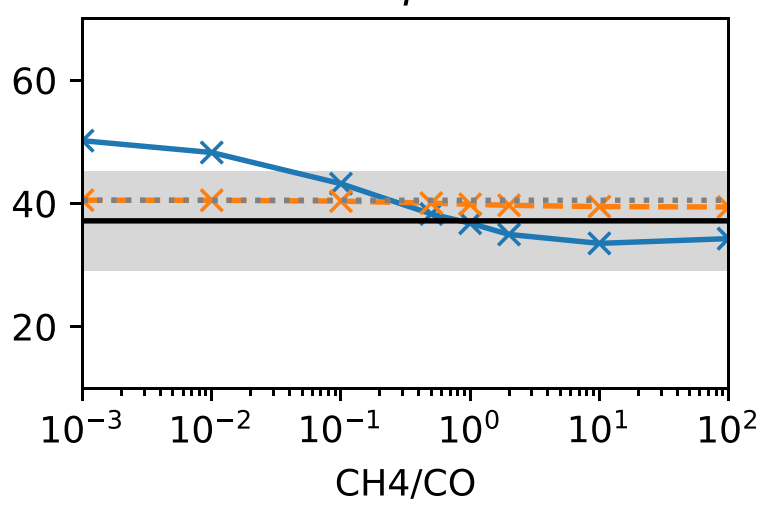

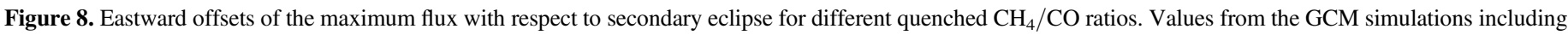

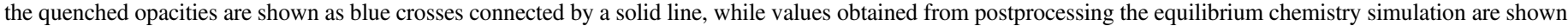

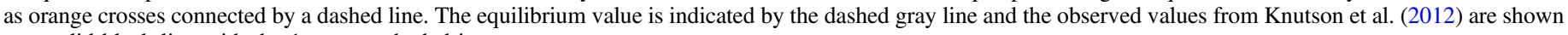
as a solid black line with the $1 \sigma$ errors shaded in gray.

and thus decrease the flux. However, they ignore that when changing the $\mathrm{CO}$ abundance through quenching, the water abundance should also change. Water has significant opacity in the $4.5 \mu \mathrm{m}$ band as well. Averaged across the band, its molecular absorption cross section is comparable to that of $\mathrm{CO}$ (see, e.g., Figure 5 in Fortney et al. 2006 or Figures 2 and 3 in Sharp \& Burrows 2007). For each $\mathrm{CO}$ molecule added, an $\mathrm{H}_{2} \mathrm{O}$ molecule is removed (assuming metallicity is held constant), and the resulting changes in opacity in the $4.5 \mu \mathrm{m}$ band due to $\mathrm{CO}$ and $\mathrm{H}_{2} \mathrm{O}$ largely offset each other (see also Section 2.3 and Figure 3). The changes in the $4.5 \mu \mathrm{m}$ phase curve are thus mainly due to the change in thermal structure and not due to a change of the photospheric level. In the hotter $\mathrm{CH}_{4}$-dominated case, the flux increases at all phase angles in this band. In the CO-dominated case, the flux near the phase-curve maximum decreases slightly compared to the equilibrium chemistry case, reflecting the cooler dayside, while the phase curve follows closely the equilibrium chemistry phase curve at other phase angles.

In the $24 \mu \mathrm{m}$ band, the difference between the disequilibrium and equilibrium chemistry simulations is relatively small. None of the predicted phase curves matches the available data on the nightside well. However, as observations in this band do not cover all phase angles, the shape of the phase curve in this band remains uncertain.

\subsection{Phase-curve Trends}

We now examine trends in the phase-curve properties with the quenched ratio. Two factors control how the phase curve in the quenched case differs from that in the equilibrium chemistry case. First, the change in opacities directly impacts the thermal structure of the planet, as discussed in Section 3.2. Second, the change in opacities causes the photospheric level to shift upward or downward. As a result, the outgoing radiation probes cooler or hotter layers. This change in the location of the photosphere can also be studied by postprocessing the output of GCM simulations assuming equilibrium chemistry with quenched opacities instead of self-consistently including quenched opacities in the GCM. It is thus of significant interest to examine the relative importance of both of these factors in shaping the predicted phase curve. If it turned out that the change in the thermal structure had only a small effect on the phase curve, modelers could restrict themselves to including disequilibrium chemistry in the postprocessing of the GCM output, which is simpler and computationally less expensive. In addition to computing the phase curves from the simulations with disequilibrium chemistry (self-consistent opacities), we thus also computed phase curves by postprocessing the output from the equilibrium chemistry simulation with quenched opacities. 


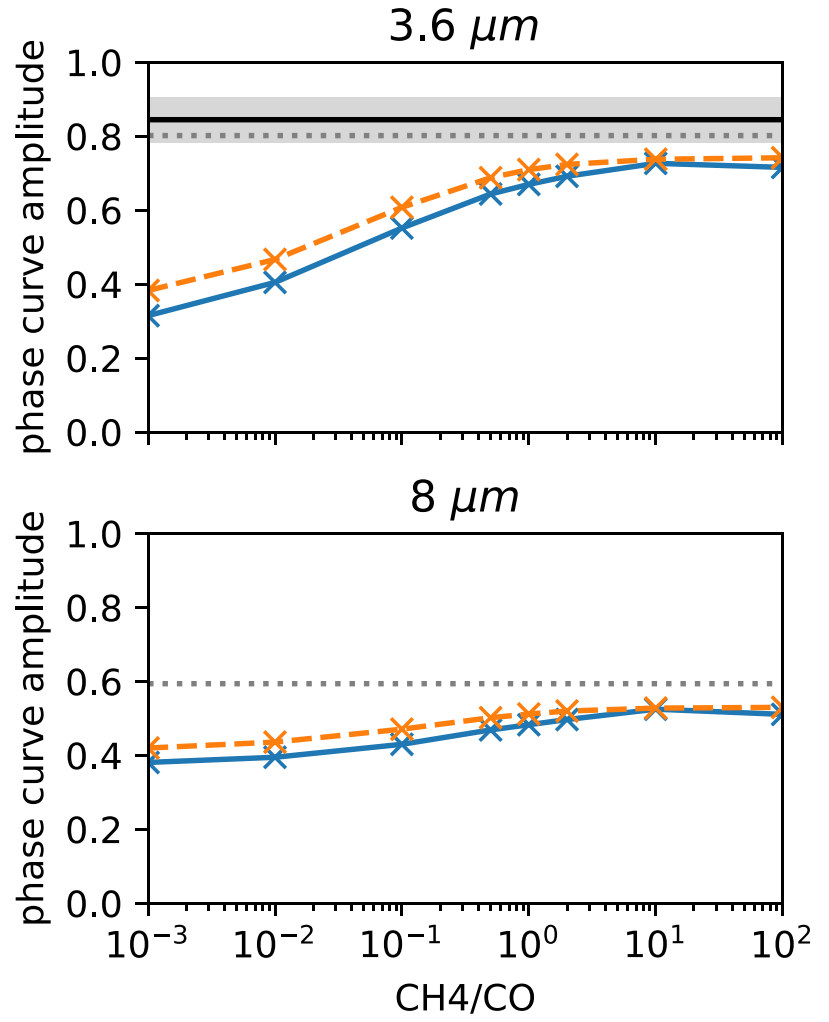

$*$ self-consistent opacities
$-*-$ postprocessed only

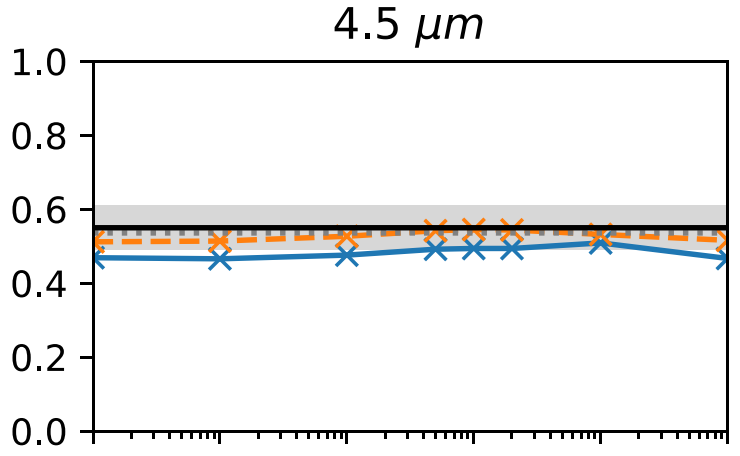

$24 \mu m$

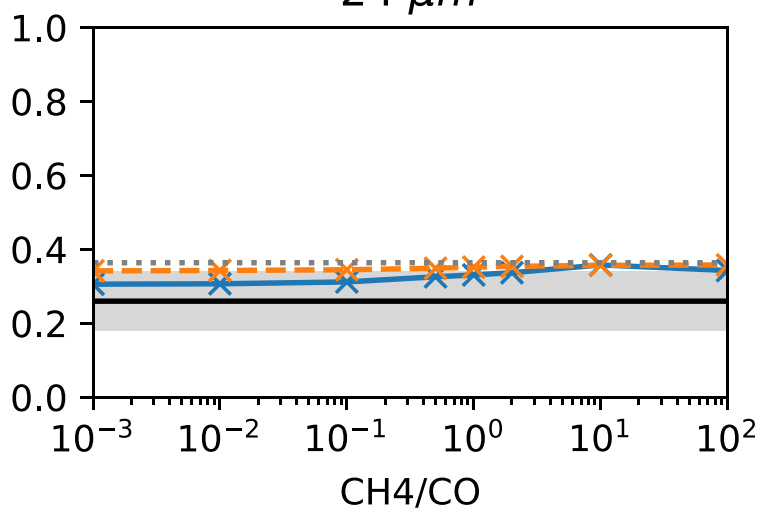

..... equilibrium value - observed value

Figure 9. Normalized phase-curve amplitudes, defined as $A=1-F_{\min } / F_{\max }$, for different quenched $\mathrm{CH}_{4} / \mathrm{CO}$ ratios. Values from the GCM simulations including the quenched opacities are shown as blue crosses connected by a solid line, while values obtained from postprocessing the equilibrium chemistry simulation are shown as orange crosses connected by a dashed line. The equilibrium value is indicated by the dashed gray line and the observed values from Knutson et al. (2012) are shown as a solid black line with the $1 \sigma$ errors shaded in gray. Note that for the $8 \mu \mathrm{m}$ band there is no observed value, because observations in this band did not cover the nightside.

Figures 7-9 show trends in secondary eclipse depth, phasecurve offset, and phase-curve amplitude, respectively, with the quenched ratio for phase curves obtained from both simulations with self-consistent opacities and from the equilibrium chemistry simulation postprocessed with quenched opacities. If both curves align, the change is dominated by the shift in the photospheric pressure. If there are large discrepancies between the postprocessed and the self-consistent GCM phase curves, the change in thermal structure plays an important role.

In the 3.6 and $8 \mu \mathrm{m}$ bands, which include strong methane absorption features, the phase-curve amplitude increases with increasing methane abundance (Figure 9), while the phasecurve offset and the secondary eclipse flux decrease (Figures 8 and 7, respectively). This is expected as the photosphere in these bands shifts to higher levels of the atmosphere with stronger day-night contrasts. All of these trends flatten once $\mathrm{CH}_{4}$ starts to dominate over $\mathrm{CO}$. The phase-curve amplitudes from the postprocessed simulations closely match the ones with self-consistent opacities, indicating that the change in the photospheric level is the dominant cause of this trend. For the phase-curve offset, the direction of the trend is similar; however, the trend is weaker in the postprocessed-only points, indicating that the change in thermal structure significantly contributes to the trend.
In the $4.5 \mu \mathrm{m}$ band, secondary eclipse flux, phase-curve offset, and phase-curve amplitude from the postprocessed-only simulations are close to the equilibrium value, indicating that the location of the photosphere changes only marginally. In the simulations with self-consistent opacities, however, the secondary eclipse flux is increasing with increased methane abundance, in line with the higher temperatures in the simulation described in Section 3.2. The phase-curve offset also decreases with increasing $\mathrm{CH}_{4}$ in the $\mathrm{CO}$-dominated regime. The phase-curve amplitudes stay similar.

In the $24 \mu \mathrm{m}$ band, there are no trends in secondary eclipse and phase-curve amplitude. For the phase-curve offset, the simulations with self-consistent opacities replicate the same decreasing trend as in other wavelength bands, as would be expected for a trend due to a change in thermal structure.

It is also of interest to examine how the trends described compare to the properties of the observed phase curve (indicated in black with gray error bars in Figures 7-9). In the 3.6 and $8 \mu \mathrm{m}$ bands, the secondary eclipse depth is closer to the observed value for low $\mathrm{CH}_{4} / \mathrm{CO}$ values than for high ones. The phase-curve offset, in contrast, matches the observed value for $\mathrm{CH}_{4} / \mathrm{CO}$ values near 0.5 in the $3.6 \mu \mathrm{m}$ band and is closest to the observed value for $\mathrm{CH}_{4} / \mathrm{CO}$ values greater than one for the 4.5 and $8 \mu \mathrm{m}$ bands. The phase-curve amplitude in the $3.6 \mu \mathrm{m}$ band is closer to the observed value for high $\mathrm{CH}_{4} / \mathrm{CO}$ 

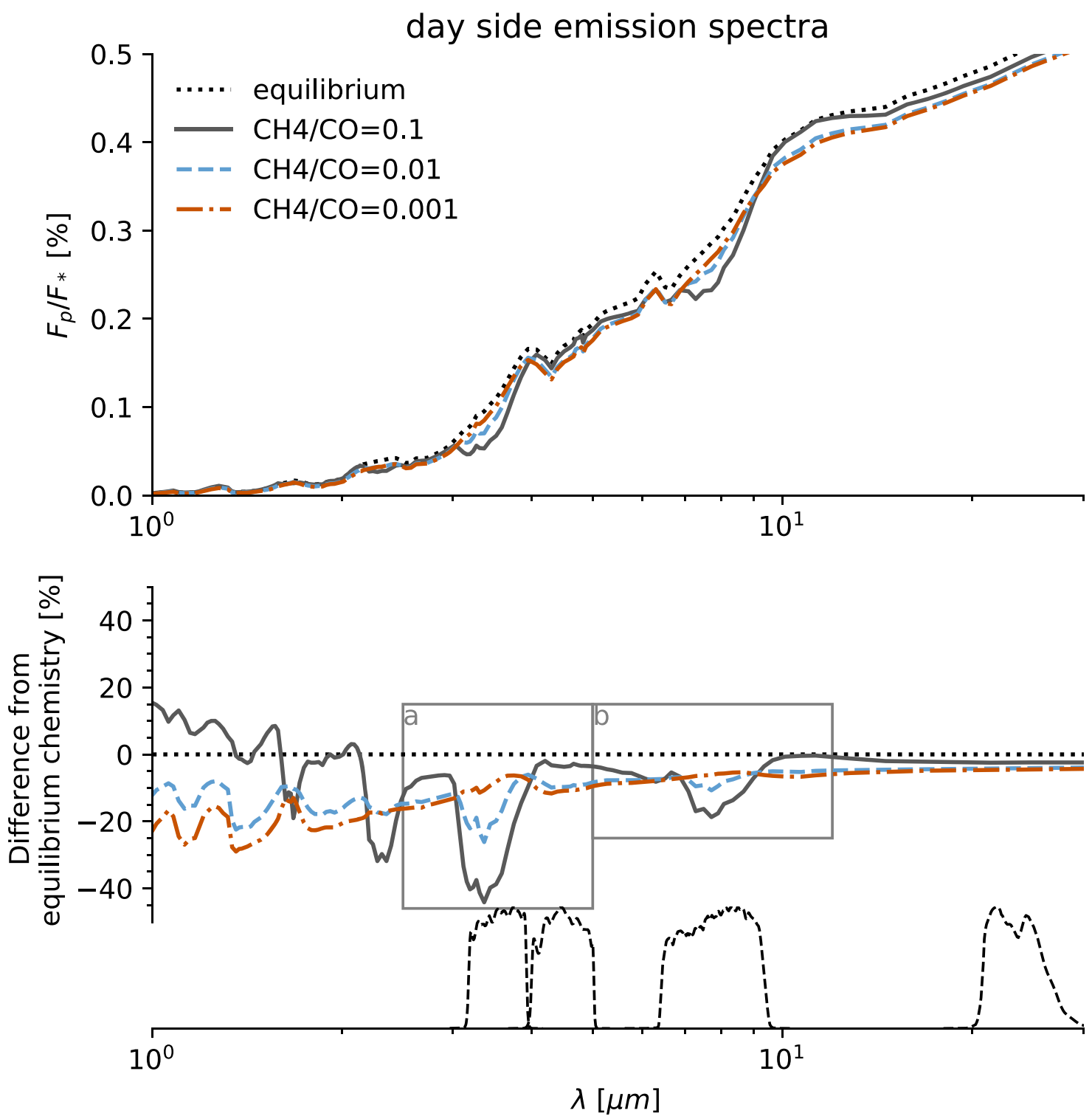

Figure 10. Predicted dayside emission spectra, as would be observed at secondary eclipse. The ratio of the planet flux to the stellar flux is plotted in the top panel. The bottom panel shows the relative difference between the flux in each of the quenched simulations and the flux in the equilibrium chemistry simulation. The regions plotted in panels a and b of Figure 12 are indicated with gray boxes. The dashed black lines at the bottom indicate the filter sensitivity profiles of the Spitzer 3.6, 4.5, 8, and $24 \mu \mathrm{m}$ bands. The emission spectra in this figure are available as Data behind the Figure. The data used to create this figure are available.

ratios. In short, among the quenched simulations there is no one $\mathrm{CH}_{4} / \mathrm{CO}$ ratio that matches the phase-curve properties best, because low $\mathrm{CH}_{4} / \mathrm{CO}$ ratios match the secondary eclipse better but high $\mathrm{CH}_{4} / \mathrm{CO}$ ratios match the phase-curve offset better. This further underlines our finding from Section 3.3 that quenching of $\mathrm{CH}_{4}$ and $\mathrm{CO}$ does not provide a good explanation for the shape of the observed phase curves.

\subsection{Emission Spectra}

To move beyond a discussion restricted to the Spitzer bands, we plot the predicted emission spectra of the dayside (Figure 10) and nightside (Figure 11) for the CO-dominated simulations. We focus on our $\mathrm{CH}_{4} / \mathrm{CO}<1$ cases because these are expected to be more likely for the temperature range of HD $189733 \mathrm{~b}$. Even though we found in the previous subsections that the disequilibrium chemistry cases from our model do not match existing observations, it is instructive to look at the effect of disequilibrium chemistry on the emission spectra. The findings could also be applied to other planets in a similar temperature regime.

In general, the dayside fluxes from the quenched simulations are lower than the fluxes from the reference case, while the nightside fluxes from the quenched simulations are significantly higher than for the reference case. This is consistent with the change in energy balance mentioned in Section 3.2.

The quenched dayside emission spectra deviate moderately from the equilibrium chemistry spectrum. The difference is largest in the water absorption bands between 1 and $2 \mu \mathrm{m}$ for the $\mathrm{CH}_{4} / \mathrm{CO}=0.001$ spectrum (up to $-30 \%$ ) and largest near the $3.3 \mu \mathrm{m}$ methane absorption band for the $\mathrm{CH}_{4} / \mathrm{CO}=0.01$ and 0.1 spectra (up to $-25 \%$ and $-40 \%$, respectively). In the $\mathrm{CH}_{4} / \mathrm{CO}=0.1$ spectrum, methane absorption bands clearly show up. 

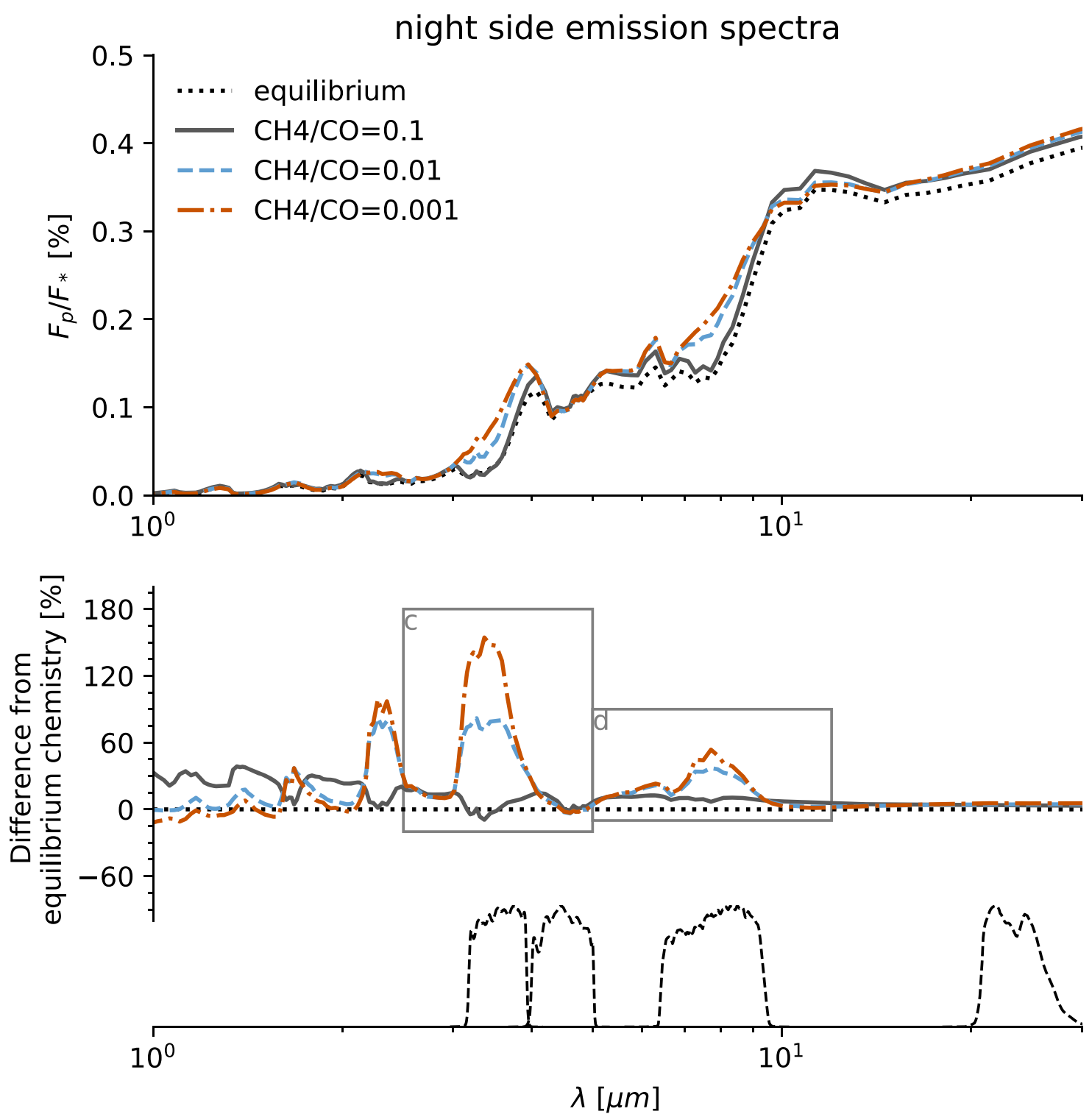

Figure 11. Same as Figure 10 but for nightside emission spectra, as would be observed just before or after transit. The emission spectra in this figure are available as Data behind the Figure. The data used to create this figure are available.

In the nightside spectra, for $\mathrm{CH}_{4} / \mathrm{CO}=0.001$ and 0.01 there are large differences compared to the equilibrium chemistry case, especially in the methane absorption bands. The largest flux difference can be found near the $3.3 \mu \mathrm{m}$ methane band (up to $160 \%$ and $80 \%$, respectively), but the $2.3 \mu \mathrm{m}$ (up to $100 \%$ and $80 \%$ ) and $7.7 \mu \mathrm{m}$ (up to $55 \%$ and $40 \%$ ) methane bands also show substantial flux differences. For the $\mathrm{CH}_{4} / \mathrm{CO}=0.1$ case, the difference from equilibrium chemistry is in general much smaller and the largest difference can be seen in the water absorption bands between 1 and $2 \mu \mathrm{m}$. In the region around $4.5 \mu \mathrm{m}$, the differences from the equilibrium chemistry case are small for all three quenched ratios, reinforcing our previous conclusion that this wavelength region is not suitable for detecting disequilibrium chemistry on the nightsides of hot Jupiters.

The fact that disequilibrium chemistry mainly affects spectral regions with $\mathrm{CH}_{4}$ bands, as well as $\mathrm{H}_{2} \mathrm{O}$ bands, raises the question of how the spectral signatures of disequilibrium chemistry can be distinguished from those of increased or decreased $\mathrm{CH}_{4}$ and $\mathrm{H}_{2} \mathrm{O}$ abundances in equilibrium chemistry due to a non-solar $\mathrm{C} / \mathrm{O}$ or $\mathrm{C} / \mathrm{H}$ ratio. While for a single secondary eclipse or nightside emission spectrum, these scenarios may look similar, we expect that the combination of a secondary eclipse and nightside emission spectrum would be able to discriminate between these scenarios.

\subsection{Simulated James Webb Space Telescope (JWST) Spectra}

In order to determine whether the $\mathrm{CH}_{4} / \mathrm{CO}$ ratio can be distinguished with future observations, we simulated JWST observations of HD 189733b using PandExo (Batalha et al. 2017). PandExo is a noise simulator that uses the Space Telescope Science Institute's exposure time calculator, Pandeia (Pontoppidan et al. 2016). Using PandExo, we find that in many NIRSpec and NIRISS observing modes the detector becomes saturated due to HD 189733b's brightness $(J=6.07)$. We therefore simulate data for two of NIRCam's grisms, F322W2 (2.413-4.083 $\mu \mathrm{m})$ and F444W (3.835-5.084 $\mu \mathrm{m})$, and 

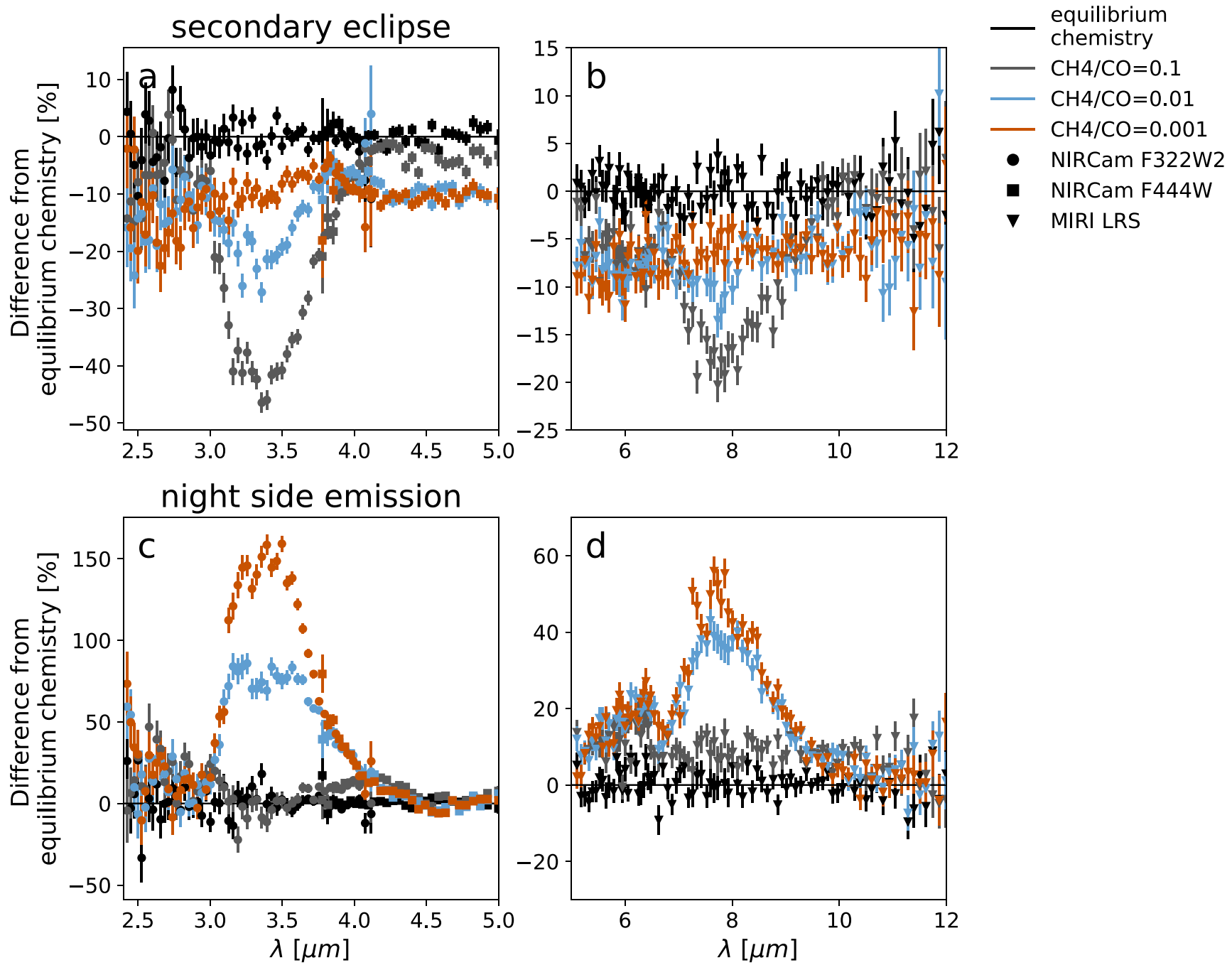

Figure 12. Simulated JWST observations for NIRCam (left panels) using the F322W2 (circles) and F444W (squares) grisms and MIRI LRS (right panels, triangles). Plotted is the difference from the flux predicted by the equilibrium chemistry model, binned to a spectral resolution of $R \approx 50$, with $1 \sigma$ error bars. The simulated observations in this figure are available as Data behind the Figure. The data used to create this figure are available.

for MIRI low-resolution spectroscopy (LRS) $(5-12 \mu \mathrm{m})$. These three modes all remain below $80 \%$ full well saturation across the detector. The NIRCam grisms utilize the $64 \times 2048$ pixel subarray to reduce readout times. MIRI LRS uses a dedicated slitless prism subarray region.

Each instrument mode was simulated assuming only a single eclipse with equal time in eclipse and out of eclipse. We also assume a noise floor of 40 parts per million, which is similar to the noise floors found in WFC3 on the Hubble Space Telescope (HST) (Kreidberg et al. 2014). We simulated data for different $\mathrm{CH}_{4} / \mathrm{CO}$ ratios and for equilibrium chemistry. Observations were simulated for both the dayside and the nightside. Since PandExo only models eclipses and transits and not phase curves, the planet's nightside spectrum was given to PandExo as if it were the planet's dayside one since the expected noise should be the same, except for the reduced brightness of the planet's nightside. We plot the results for the CO-dominated simulations in Figure 12 in terms of the difference from the equilibrium chemistry model. We also include the simulated observations for the equilibrium chemistry case to give a sense of the expected residuals. Note that the scale on the $y$-axis is different in each panel. For better orientation, the regions shown in each panel are indicated with gray boxes in Figures 10 and 11.

In secondary eclipse, all quenched cases can be distinguished from the disequilibrium chemistry case in all three observing modes. The different $\mathrm{CH}_{4} / \mathrm{CO}$ ratios of $0.1,0.01$, and 0.001 can also clearly be distinguished from each other. With MIRI LRS, the fluxes in the $\mathrm{CH}_{4} / \mathrm{CO}=0.01$ and 0.001 cases are relatively close to each other, but can still be distinguished from each other at greater than $3 \sigma$ confidence.

Looking at nightside emission spectra, the relative deviations from the equilibrium chemistry spectra are much larger. The $\mathrm{CH}_{4} / \mathrm{CO}=0.01$ and 0.001 cases can easily be distinguished from equilibrium chemistry in all three observing modes. The nightside fluxes of the $\mathrm{CH}_{4} / \mathrm{CO}=0.1$ case are in general much closer to the equilibrium chemistry case in the wavelength ranges covered by these observing modes, but still can be distinguished from disequilibrium chemistry. With the NIRCam F322W2 grism and MIRI LRS, it is possible to clearly distinguish the three $\mathrm{CH}_{4} / \mathrm{CO}$ ratios shown from each other as well. With simulated data from the NIRCam F444W grism, it is still possible to tell the different $\mathrm{CH}_{4} / \mathrm{CO}$ ratios apart from each other at $3 \sigma$ confidence, but differences between 
the quenched ratios are only evident at wavelengths shorter than $4 \mu \mathrm{m}$, at the very edge of the wavelength range of this grism. Thus, it may not be the first choice for observing disequilibrium chemistry.

Our intent in presenting these simulated observations is to demonstrate the need to include disequilibrium chemistry when interpreting future observations with JWST and to guide observers in which regions to look for signatures of disequilibrium carbon chemistry. However, it would be premature to conclude that one would be able to determine the $\mathrm{CH}_{4} / \mathrm{CO}$ ratio, because other factors not considered in this work can influence the results of GCM simulations and the emission spectra, including clouds, metallicity, $\mathrm{C} / \mathrm{O}$ ratio, and atmospheric drag (see Section 4.2). In particular, an optically thick cloud deck on the nightside could limit our ability to observe signatures of disequilibrium chemistry, because it would block emission from the layers in the atmosphere below the cloud deck. This could mute the strongly visible $\mathrm{CH}_{4}$ features in Figures 10-12 in which the difference from equilibrium chemistry is most obvious. Future work in this direction is necessary.

\section{Discussion}

\subsection{Importance of Including Disequilibrium Chemistry in GCMs}

In their study of HD 209458b, Drummond et al. (2018b) find that the effect of transport-induced disequilibrium chemistry on the temperature structure and winds is $\sim 1 \%$ relative to otherwise similar models with chemical equilibrium. In contrast, in our simulations of HD 189733b we find an effect on the temperature of up to $\sim 5 \%-10 \%$ across large regions (larger changes in very localized regions) in the more likely $\mathrm{CO}$-dominated regime and even larger temperature changes in the $\mathrm{CH}_{4}$-dominated regime. While this paper was in review, Drummond et al. (2018a) found a similar result in their simulations of HD 189733b. This raises the question of why this effect is so much larger on HD 189733b. The answer lies in the lower temperature of HD 189733b. On the hotter HD $209458 \mathrm{~b}$ (equilibrium temperature $T_{\mathrm{eq}} \approx 1450 \mathrm{~K}$ ), CO remains the dominant species even on the nightside in both equilibrium and disequilibrium chemistry. The change in opacity due to disequilibrium chemistry is thus less drastic. In contrast, on the cooler HD $189733 \mathrm{~b}\left(T_{\mathrm{eq}} \approx 1200 \mathrm{~K}\right)$ the nightside is cold enough that with equilibrium chemistry $\mathrm{CH}_{4}$ becomes the dominant species on a significant fraction of the nightside. In disequilibrium chemistry, however, $\mathrm{CO}$ is expected to be the dominant species everywhere at pressures below 1 bar. Thus, when including disequilibrium chemistry in the radiative transfer, for most of the nightside the dominant carbon species switches from $\mathrm{CH}_{4}$ to $\mathrm{CO}$, significantly changing the opacities. With this switch between $\mathrm{CH}_{4}$ and $\mathrm{CO}$ as dominant species, the $\mathrm{H}_{2} \mathrm{O}$ abundance changes by a factor of about two as well, further affecting the opacities. For planets much cooler than HD 189733b, even the dayside becomes $\mathrm{CH}_{4}$-dominated in both equilibrium and disequilibrium chemistry. Regardless of exact disequilibrium abundances, the dominant species remains the same as in equilibrium chemistry on these planets. For this situation, we would thus again expect a smaller effect on the opacities and thus on the resulting temperatures.

Therefore, we expect that including the effect of disequilibrium carbon chemistry on the opacities in the GCM is most important for planets for which equilibrium chemistry predicts a CO-dominated dayside but a $\mathrm{CH}_{4}$-dominated nightside. Assuming solar metallicity, the equilibrium abundance of $\mathrm{CH}_{4}$ becomes comparable to the $\mathrm{CO}$ abundance on the coldest regions of the nightside for planets with equilibrium temperatures below $\sim 1300 \mathrm{~K}$ (Kataria et al. 2016, Figure 7, taking the morning terminator profiles to be representative of the coldest regions of the planet). This demarcates the upper boundary of the regime in which we expect disequilibrium carbon abundances to be important in GCMs. The colder end of this regime has been less explored by GCMs. Based on 1D models, for solar composition the dayside is expected to transition from CO-dominated to $\mathrm{CH}_{4}$-dominated roughly at equilibrium temperatures around $700 \mathrm{~K}$, with the transition occurring at slightly higher temperatures in equilibrium chemistry and at slightly lower ones in disequilibrium chemistry (Moses et al. 2013a; Miguel \& Kaltenegger 2014; Venot et al. 2014). We thus expect that it is important to include disequilibrium $\mathrm{CH}_{4}$ and $\mathrm{CO}$ abundances in GCMs of hot Jupiters for planets with equilibrium temperatures between $\sim 600$ and $1300 \mathrm{~K}$, assuming solar abundances. As the temperature of the transition between $\mathrm{CH}_{4}$ and $\mathrm{CO}$ depends strongly on the metallicity and $\mathrm{C} / \mathrm{O}$ ratio, this temperature range may change depending on atmospheric composition. Future work spanning a range of planets is necessary to test this prediction. This could include both simulations coupling a chemical scheme to a GCM similar to Drummond et al. (2018b) and Cooper \& Showman (2006) and simulations with a simpler approach comparable to ours.

While we expect these findings to qualitatively also apply to atmospheres with moderate deviations from solar metallicity and $\mathrm{C} / \mathrm{O}$ ratio, our conclusions may not apply to planets with $\mathrm{C} / \mathrm{O}$ ratios $>1$. In that case, the composition changes drastically and the abundances of $\mathrm{CH}_{4}$ and $\mathrm{HCN}$ can become comparable to that of $\mathrm{CO}$ for both equilibrium chemistry and disequilibrium chemistry even on the daysides of planets with temperatures comparable to HD 189733b or hotter (Moses et al. 2013b). However, to date no planet atmosphere has been shown to have a $\mathrm{C} / \mathrm{O}$ ratio $>1$ (Line et al. 2014; Benneke 2015).

We further note that our method of assuming a constant $\mathrm{CH}_{4} / \mathrm{CO}$ ratio is only a good approximation for situations in which $\mathrm{CH}_{4}$ and $\mathrm{CO}$ abundances have been homogenized horizontally and vertically throughout most of the photosphere (at pressures between $\sim 1$ mbar and 1 bar). While for hot Jupiters with relatively low equilibrium temperatures, such as HD 189733b, this is expected to be the case (Cooper \& Showman 2006; Agúndez et al. 2014; Drummond et al. 2018b), there can be situations in which abundances are homogenized only horizontally and not vertically, or in which the quenching happens only at lower pressures and significant parts of the photosphere are still in chemical equilibrium (Agúndez et al. 2014; Mendonça et al. 2018a). In general, such situations are expected for planets with higher dayside temperatures and weak vertical mixing. Future work is necessary to better understand when such situations occur and to clarify the relative importance of horizontal and vertical quenching.

\subsection{Other Factors Impacting the Predicted Phase Curves}

As discussed in Section 3.3, disequilibrium carbon chemistry barely affects the $4.5 \mu \mathrm{m}$ phase curve while significantly decreasing the phase-curve amplitude in the $3.6 \mu \mathrm{m}$ band, thus 
worsening the fit of the $3.6 \mu \mathrm{m}$ phase curve to observations. Among the simulations we ran for this paper, the equilibrium chemistry simulation actually matches the data better than any of the disequilibrium chemistry simulations. At this point, we would like to note that the equilibrium chemistry phase curve in this paper somewhat differs from the solar-metallicity (equilibrium chemistry) phase curve presented in Showman et al. (2009) and Knutson et al. (2012). This is due to updates of the opacities (Freedman et al. 2014) and planetary and stellar parameters. In general, in all wavelength bands, the phasecurve amplitude increases and the phase-curve offset decreases slightly with the updated opacities. With these changes, the issue of the model overpredicting the nightside fluxes in the $4.5 \mu \mathrm{m}$ band is somewhat mitigated and difference in phasecurve offset becomes the more dominant discrepancy between observations and the solar-metallicity equilibrium chemistry model.

Our finding that the phase curve from the equilibrium chemistry simulation matches the observational phase curve better than the ones assuming disequilibrium chemistry is in agreement with Dobbs-Dixon \& Cowan (2017), who find that based on their GCM the phase-curve data are consistent with equilibrium chemistry. Does this mean that the atmosphere of HD $189733 \mathrm{~b}$ is in chemical equilibrium? Based on the theoretical understanding of chemical kinetics and atmospheric dynamics, this is highly unlikely. It is much more likely that other processes that our model does not take into account are responsible for the shape of the phase curve. A likely explanation is the presence of clouds on the nightside of HD 189733b. Cloud microphysics models (Lee et al. 2015; Powell et al. 2018) and GCM simulations including clouds with varying levels of complexity (e.g., Lee et al. 2016; Oreshenko et al. 2016; Parmentier et al. 2016; Lines et al. 2018) show that in the atmospheres of hot Jupiters there exist a variety of species that may condense to form clouds on the nightside. An optically thick cloud deck on the nightside would block the emission from hotter, deeper layers in the atmosphere, thus reducing the flux emitted on the nightside over a broad range of wavelengths. Clouds present only on the nightside of the planet would therefore increase the amplitude of the phase curve. Mendonça et al. (2018a) include a simple parameterization of nightside clouds in their GCM simulations of WASP-43b and study the effect on the spectra and phase curves. They are able to match the spectrally resolved HST WFC3 phase curve from 1.1 to $1.7 \mu \mathrm{m}$ and the Spitzer $3.6 \mu \mathrm{m}$ phase curve quite naturally. This demonstrates both the importance of including the effect of nightside clouds and the potential of clouds to explain the low observed nightside fluxes on several hot Jupiters. However, the effect of clouds on spectra depends on many unknown properties such as cloud top pressure, composition, and latitudinal and longitudinal distribution, and more theoretical and observational work is necessary. Furthermore, to match the $4.5 \mu \mathrm{m}$ phase curve, Mendonça et al. (2018a) include additional $\mathrm{CO}_{2}$ compared to the equilibrium chemistry abundance on the nightside. There is no clear theoretical motivation for additional $\mathrm{CO}_{2}$ on the nightside-in contrast, Agúndez et al. (2014) find that disequilibrium chemistry tends to reduce the $\mathrm{CO}_{2}$ abundance on the nightsides of HD 209458b and HD 189733b compared to equilibrium chemistry. Mendonça et al. (2018b) find that disequilibrium chemistry reduces the $\mathrm{CO}_{2}$ abundance on the nightside in the $\mathrm{C} / \mathrm{O}=0.5$ case but increases it in the $\mathrm{C} / \mathrm{O}=2$ case. They conclude that the changes in $\mathrm{CO}_{2}$ due to disequilibrium chemistry cannot resolve the remaining discrepancy in the $4.5 \mu \mathrm{m}$ band.

Especially on relatively cool planets such as HD 189733b, clouds need not be restricted to the nightside. An optically thick cloud deck extending into parts of the dayside or even covering the entire planet is another plausible possibility (e.g., Parmentier et al. 2016; Roman \& Rauscher 2019). Using a simple cloud scheme with constant particle size that includes radiative feedback, Roman \& Rauscher (2019) find that their cloud distribution (covering large fractions of the dayside) results in a larger phase amplitude and lower phase offset than in the cloud-free case. They stress the role of cloud radiative feedback in shaping the phase curve. However, it is likely that a realistic distribution of clouds over the planets is not uniform in particle size and density (Lee et al. 2016; Lines et al. 2018), potentially resulting in a much more complex effect on the phase curve (Lines et al. 2018).

In addition to clouds, several other parameters not explored in this study can significantly impact the phase curves, including non-solar metallicities and $\mathrm{C} / \mathrm{O}$ ratios, and atmospheric drag due to sub-grid-scale turbulence or due to Lorentz forces in a partially ionized atmosphere. Independent of these factors, there are also moderate uncertainties associated with the numerical model, most notably the uncertainty in the opacities of some species, which are not well known at high temperatures. In this context, we would like to remind the reader that the simulations presented are pure forward models and we make no attempt to fit the observational data. The general trends observed in this paper thus are much more important and meaningful than specific predictions for the emitted flux or other observable quantities.

\section{Conclusion}

We have included the radiative effect of transport-induced disequilibrium $\mathrm{CH}_{4}, \mathrm{CO}$, and $\mathrm{H}_{2} \mathrm{O}$ abundances in a GCM to study the effect on the atmospheric structure, phase curves, and emission spectra. We have assumed that the ratio of $\mathrm{CH}_{4}$ to $\mathrm{CO}$ is constant throughout the entire simulation (an assumption that is expected to be well fulfilled at pressures between $\sim 10^{-4}$ bars and 1 bar) and treat the $\mathrm{CH}_{4} / \mathrm{CO}$ ratio as a free parameter. The water abundance is updated accordingly, such that the total number of oxygen atoms is preserved. It is important to include this change in the water abundance, because in equilibrium chemistry the water abundance varies by a factor of $\sim 2$ between the $\mathrm{CO}$-dominated dayside and the $\mathrm{CH}_{4}$-dominated nightside. Assuming vertical and horizontal quenching, however, the water abundance is expected to be homogenized between dayside and nightside. We ran simulations of hot Jupiter HD 189733 b with eight different quenched $\mathrm{CH}_{4} / \mathrm{CO}$ ratios.

We find that in the CO-dominated case, which is the case favored by chemical kinetics models, the temperature changes locally by up to $150 \mathrm{~K}$, with lower temperatures than equilibrium chemistry on the dayside and higher temperatures on part of the nightside. In the less plausible $\mathrm{CH}_{4}$-dominated case, the addition of greenhouse gases leads to higher temperatures everywhere at pressures higher than a few tens of millibars. When comparing the phase curves predicted by GCM simulations that include disequilibrium $\mathrm{CH}_{4}$ and $\mathrm{CO}$ abundances to phase curves obtained from an equilibrium chemistry GCM simulation that has been postprocessed 
assuming quenched $\mathrm{CH}_{4} / \mathrm{CO}$ abundances, we find that the eastward offset of the phase-curve maximum can differ by up to $10^{\circ}$

We thus conclude that it is important to self-consistently include the effect of disequilibrium abundances of $\mathrm{CH}_{4}$ and $\mathrm{CO}$ on the opacities in GCMs rather than to include disequilibrium abundances only in the postprocessing while continuing to use opacities based on equilibrium chemistry abundances in the GCM. This is in contrast to Drummond et al. (2018b), who find in their study of HD 209458b that the effect of radiative feedback of disequilibrium abundances on the temperature and wind fields is only $\sim 1 \%$, but agrees with their more recent findings for HD 189733b (Drummond et al. 2018a). These seemingly conflicting results can be understood when considering the difference in the equilibrium temperatures of the planets: on the hotter HD 209458b, the $\mathrm{CH}_{4}$ abundance remains low compared to the $\mathrm{CO}$ and $\mathrm{H}_{2} \mathrm{O}$ abundances in both equilibrium and disequilibrium chemistry even on the nightside. In contrast, on the cooler HD 189733b, the nightside is cool enough to be dominated by $\mathrm{CH}_{4}$ in equilibrium chemistry. Including disequilibrium chemistry thus changes the dominant carbon species on half of the planet, resulting in much larger changes. In addition, in the regions where disequilibrium chemistry changes the dominant carbon species, the water abundance is also altered by a factor of $\sim 2$, further contributing to the effect on temperatures.

Furthermore, we show that disequilibrium $\mathrm{CH}_{4}$ and $\mathrm{CO}$ abundances have only a small effect on the Spitzer $4.5 \mu \mathrm{m}$ phase curve despite $\mathrm{CO}$ having a prominent absorption band within this wavelength band. This is because the change in opacity due to $\mathrm{CO}$ is offset by a change in water opacity in the opposite direction. In wavelength regions dominated by $\mathrm{CH}_{4}$ opacity, including the Spitzer 3.6 and $8 \mu \mathrm{m}$ bands, the phasecurve amplitude decreases significantly, resulting in a much worse fit to the observed Spitzer 3.6 and $8 \mu \mathrm{m}$ phase curves. We thus conclude that disequilibrium carbon chemistry cannot explain the observed low nightside fluxes in the $4.5 \mu \mathrm{m}$ band, in contrast to the interpretation of Knutson et al. (2012). Other effects, for example nightside clouds, must be responsible for the observed shape of the phase curve.

While disequilibrium chemistry does not explain existing observations of HD 189733b, it may be detectable on other hot Jupiters with a similar equilibrium temperature. Therefore, we examine the effect of disequilibrium carbon chemistry on emission spectra and simulated JWST observations. We find that in the expected $\mathrm{CO}$-dominated regime, spectral regions dominated by methane absorption bands are most suitable to observe disequilibrium abundances. Assuming that the spectral signatures of disequilibrium carbon chemistry are not obscured by clouds or other effects not considered in our model, it will be possible to distinguish between different quenched ratios with JWST in both secondary eclipse and phase-curve observations.

We thank Jonathan Fortney and Thaddeus Komacek for helpful comments and Heather Knutson for sharing the observational data for Figure 6. This research was supported in part by NASA Origins grant NNX12AI79G to A.P.S. and by NASA Headquarters under the NASA Earth and Space Science Fellowship Program-Grant 80NSSC18K1248. This work benefited from the Exoplanet Summer Program in the Other Worlds Laboratory (OWL) at the University of California,
Santa Cruz, a program funded by the Heising-Simons Foundation.

\section{ORCID iDs}

Maria E. Steinrueck (10 https://orcid.org/0000-0001-8342-1895 Vivien Parmentier (i) https://orcid.org/0000-0001-9521-6258 Joshua D. Lothringer (iD https://orcid.org/0000-00033667-8633

Roxana E. Lupu (iD https://orcid.org/0000-0003-3444-5908

\section{References}

Adcroft, A., Campin, J.-M., Hill, C., \& Marshall, J. 2004, MWRv, 132, 2845 Agol, E., Cowan, N. B., Knutson, H. A., et al. 2010, ApJ, 721, 1861

Agúndez, M., Parmentier, V., Venot, O., Hersant, F., \& Selsis, F. 2014, A\&A, 564, A73

Agúndez, M., Venot, O., Iro, N., et al. 2012, A\&A, 548, A73

Amundsen, D. S., Mayne, N. J., Baraffe, I., et al. 2016, A\&A, 595, A36

Amundsen, D. S., Tremblin, P., Manners, J., Baraffe, I., \& Mayne, N. J. 2017, A\&A, 598, A97

Batalha, N. E., Mandell, A., Pontoppidan, K., et al. 2017, PASP, 129, 064501 Benneke, B. 2015, arXiv:1504.07655

Boyajian, T., von Braun, K., Feiden, G. A., et al. 2015, MNRAS, 447, 846

Burrows, A., Marley, M., Hubbard, W. B., et al. 1997, ApJ, 491, 856

Burrows, A., Sudarsky, D., \& Hubbard, W. B. 2003, ApJ, 594, 545

Cooper, C. S., \& Showman, A. P. 2006, ApJ, 649, 1048

Dobbs-Dixon, I., \& Agol, E. 2013, MNRAS, 435, 3159

Dobbs-Dixon, I., \& Cowan, N. B. 2017, ApJL, 851, L26

Dobbs-Dixon, I., \& Lin, D. N. C. 2008, ApJ, 673, 513

Drummond, B., Mayne, N. J., Manners, J., et al. 2018a, ApJ, 869, 28

Drummond, B., Mayne, N. J., Manners, J., et al. 2018b, ApJL, 855, L31

Drummond, B., Tremblin, P., Baraffe, I., et al. 2016, A\&A, 594, A69

Fortney, J. J., Cooper, C. S., Showman, A. P., Marley, M. S., \& Freedman, R. S. 2006, ApJ, 652, 746

Fortney, J. J., Lodders, K., Marley, M. S., \& Freedman, R. S. 2008, ApJ, 678,1419

Fortney, J. J., Marley, M. S., Lodders, K., Saumon, D., \& Freedman, R. 2005, ApJL, 627, L69

Freedman, R. S., Lustig-Yaeger, J., Fortney, J. J., et al. 2014, ApJS, 214, 25

Freedman, R. S., Marley, M. S., \& Lodders, K. 2008, ApJS, 174, 504

Goody, R. M., \& Yung, Y. L. 1989, Atmospheric Radiation: Theoretical Basis (Oxford: Oxford Univ. Press)

Guillot, T., \& Showman, A. P. 2002, A\&A, 385, 156

Hauschildt, P. H., Allard, F., \& Baron, E. 1999, ApJ, 512, 377

Heng, K., Frierson, D. M. W., \& Phillipps, P. J. 2011, MNRAS, 418, 2669

Kataria, T., Showman, A. P., Fortney, J. J., et al. 2015, ApJ, 801, 86

Kataria, T., Showman, A. P., Fortney, J. J., Marley, M. S., \& Freedman, R. S. 2014, ApJ, 785, 92

Kataria, T., Showman, A. P., Lewis, N. K., et al. 2013, ApJ, 767, 76

Kataria, T., Sing, D. K., Lewis, N. K., et al. 2016, ApJ, 821, 9

Knutson, H. A., Charbonneau, D., Allen, L. E., et al. 2007, Natur, 447, 183

Knutson, H. A., Charbonneau, D., Cowan, N. B., et al. 2009, ApJ, 690, 822

Knutson, H. A., Lewis, N., Fortney, J. J., et al. 2012, ApJ, 754, 22

Komacek, T. D., \& Youdin, A. N. 2017, ApJ, 844, 94

Kreidberg, L., Bean, J. L., Désert, J.-M., et al. 2014, Natur, 505, 69

Lee, G., Dobbs-Dixon, I., Helling, C., Bognar, K., \& Woitke, P. 2016, A\&A, 594, A48

Lee, G., Helling, C., Dobbs-Dixon, I., \& Juncher, D. 2015, A\&A, 580, A12 Lewis, N. K., Showman, A. P., Fortney, J. J., et al. 2010, ApJ, 720, 344

Lewis, N. K., Showman, A. P., Fortney, J. J., Knutson, H. A., \& Marley, M. S. 2014, ApJ, 795, 150

Line, M. R., Knutson, H., Wolf, A. S., \& Yung, Y. L. 2014, ApJ, 783, 70

Lines, S., Mayne, N. J., Boutle, I. A., et al. 2018, A\&A, 615, A97

Lodders, K., \& Fegley, B. 2002, Icar, 155, 393

Marley, M. S., \& McKay, C. P. 1999, Icar, 138, 268

Marley, M. S., Saumon, D., Guillot, T., et al. 1996, Sci, 272, 1919

Marley, M. S., Seager, S., Saumon, D., et al. 2002, ApJ, 568, 335

Mayne, N. J., Baraffe, I., Acreman, D. M., et al. 2014, A\&A, 561, A1

McKay, C. P., Pollack, J. B., \& Courtin, R. 1989, Icar, 80, 23

Mendonça, J. M., Grimm, S. L., Grosheintz, L., \& Heng, K. 2016, ApJ, 829,115

Mendonça, J. M., Malik, M., Demory, B.-O., \& Heng, K. 2018a, AJ, 155, 150 
Mendonça, J. M., Tsai, S.-m., Malik, M., Grimm, S. L., \& Heng, K. 2018b, ApJ, 869, 107

Menou, K. 2019, MNRAS, 485, L98

Miguel, Y., \& Kaltenegger, L. 2014, ApJ, 780, 166

Moses, J. I., Line, M. R., Visscher, C., et al. 2013a, ApJ, 777, 34

Moses, J. I., Madhusudhan, N., Visscher, C., \& Freedman, R. S. 2013b, ApJ, 763,25

Moses, J. I., Visscher, C., Fortney, J. J., et al. 2011, ApJ, 737, 15

Oreshenko, M., Heng, K., \& Demory, B.-O. 2016, MNRAS, 457, 3420

Parmentier, V., Fortney, J. J., Showman, A. P., Morley, C., \& Marley, M. S. 2016, ApJ, 828, 22

Parmentier, V., Line, M. R., Bean, J. L., et al. 2018, A\&A, 617, A110

Parmentier, V., Showman, A. P., \& Lian, Y. 2013, A\&A, 558, A91

Perna, R., Heng, K., \& Pont, F. 2012, ApJ, 751, 59

Pontoppidan, K. M., Pickering, T. E., Laidler, V. G., et al. 2016, Proc. SPIE, 9910, 991016

Powell, D., Zhang, X., Gao, P., \& Parmentier, V. 2018, ApJ, 860, 18

Rauscher, E., \& Menou, K. 2012, ApJ, 750, 96

Roman, M., \& Rauscher, E. 2019, ApJ, 872, 1
Shapiro, R. 1970, RvGSP, 8, 359

Sharp, C. M., \& Burrows, A. 2007, ApJS, 168, 140

Showman, A. P., Fortney, J. J., Lewis, N. K., \& Shabram, M. 2013, ApJ, 762,24

Showman, A. P., Fortney, J. J., Lian, Y., et al. 2009, ApJ, 699, 564

Showman, A. P., \& Guillot, T. 2002, A\&A, 385, 166

Showman, A. P., Lewis, N. K., \& Fortney, J. J. 2015, ApJ, 801, 95

Showman, A. P., \& Polvani, L. M. 2011, ApJ, 738, 71

Stevenson, K. B., Line, M. R., Bean, J. L., et al. 2017, AJ, 153, 68

Thrastarson, H. T., \& Cho, J. Y.-K. 2011, ApJ, 729, 117

Toon, O. B., McKay, C. P., Ackerman, T. P., \& Santhanam, K. 1989, JGR, 94, 16287

Tremblin, P., Chabrier, G., Mayne, N. J., et al. 2017, ApJ, 841, 30

Tsai, S.-M., Kitzmann, D., Lyons, J. R., et al. 2018, ApJ, 862, 31

Venot, O., Agúndez, M., Selsis, F., Tessenyi, M., \& Iro, N. 2014, A\&A, 562, A51

Visscher, C., Lodders, K., \& Fegley, B., Jr. 2006, ApJ, 648, 1181

Visscher, C., \& Moses, J. I. 2011, ApJ, 738, 72

Zellem, R. T., Lewis, N. K., Knutson, H. A., et al. 2014, ApJ, 790, 53 